\title{
A Decomposition Method for Synthesizing Complex Column Configurations Using Tray-by-Tray GDP Models
}

\author{
Mariana Barttfeld, Pío A. Aguirre* and Ignacio E. Grossmann ${ }^{* *}$ \\ * INGAR- Instituto de Desarrollo y Diseño - Avellaneda 3657- (3000) Santa Fe, Argentina. \\ ** Department of Chemical Engineering, Carnegie Mellon University - Pittsburgh, PA 15213, USA. \\ grossmann@cmu.edu
}

Keywords: disjunctive programming, complex distillation columns, initialization, discrete decision.

\begin{abstract}
This paper describes an optimization procedure for the synthesis of complex distillation configurations. A superstructure based on the Reversible Distillation Sequence Model (RDSM) is proposed embedding all possible alternative designs using tray-by-tray models. Generalized Disjunctive Programming (GDP) is used to model the superstructure. Due to the large size and complexity of the formulation, a decomposition solution strategy is proposed where discrete decisions are decomposed into two hierarchical levels within an iterative procedure. In the first level, the column sections are selected yielding a candidate configuration. In the second level, the feed location and the number of trays of the selected sections are optimized. A preprocessing phase including thermodynamic information is considered to provide a good starting point to the algorithm in order to improve the convergence and robustness of the method. Examples are presented for zeotropic and azeotropic multicomponent mixtures to illustrate the performance of the proposed method. Nontrivial configurations are obtained involving modest solution times.
\end{abstract}

\section{Introduction}

The synthesis of a process addresses the fundamental problem of finding a configuration that satisfies a set of goals and specifications. The separation of more than two components by continuous distillation has been usually accomplished by arranging columns in series. However, even under the assumption of minimum reflux, past work has shown that complex arrangements can lead to significant savings in the operating costs. Most of the effort in the field of distillation synthesis has been applied to develop short-cut and simplified methods (Fidkowski and Krolikowski, 1986; Glinos and Malone, 1988; Triantafyllou and Smith, 1992; Annakou and Mizsey, 1996). As an example of recent work, Caballero and Grossmann (2002) have 
presented a systematic approach for generating all the thermodynamic equivalent structures for a given sequence.

The generation of complex column configurations has been principally developed by Sargent and Gaminibandara (1976), Agrawal (1996) and Fidkowski and Agrawal (1995, 1996). Other superstructures include for instance the one by Koehler et al. (1992) who considered thermodynamic aspects. However, the problem of systematically obtaining the optimal design out of superstructure was not addressed by these authors. Some recent work has applied mathematical programming tools to rigorously solve the distillation design problem. The superstructure most commonly used in the literature is based on the one proposed by Sargent and Gaminibandara for ideal mixtures (1976) and later extended for azeotropic cases (Sargent, 1998). A different superstructure that is not so commonly used is the one proposed by Bauer and Stichlmair (1995) that uses thermodynamic information in the representation itself. These authors applied this representation in the design of azeotropic sequences.

Dunnebier and Pantelides (1999) have considered the optimal design of thermally coupled distillation columns and dividing wall columns for ideal mixtures using detailed distillation models and optimization. The solution of these problems is non-trivial since they are solved in full space. Yeomans and Grossmann (2000a) developed a disjunctive programming procedure for the optimal design of ideal and nonideal trayby-tray distillation units and separation sequences as well as complex column configurations (Yeomans and Grossmann, 2000b). While in these methods the size of the NLP subproblems is reduced the optimization of superstructures is also non-trivial due to the nonlinearities and nonconvexities inherent in theses problems.

Based on our previous work (Barttfeld et al., 2003) where alternative representation and formulations for single distillation columns were studied, a general superstructure is presented in this paper. A new generalized disjunctive optimization model for the synthesis of complex columns configurations is then proposed as well as a new effective decomposition algorithm for optimizing the superstructure. Also, a preprocessing procedure is included as the initialization phase in order to increase the robustness of the proposed algorithm. The major motivation in this work is to produce a method that is robust and has reasonable computation requirements.

This paper is organized as follows. In Section 2 the problem is stated. In Section 3, the solution strategy used to model and solve the complex columns configurations is outlined. The superstructure is described in Section 4, while in Section 5, the detailed formulations are presented. In Section 6, the detailed solution procedure is described. In Section 7, several examples are produced to illustrate the performance of the proposed synthesis method. Finally, results and conclusions are discussed in Section 8. 


\section{Problem Statement}

The problem addressed in this paper can be stated as follows. Given is a multicomponent feed with known flowrate and composition, and given are the desired products specifications. The problem then consists in selecting the structure and operating conditions of a complex configuration of distillation columns involving minimum investment and operating cost. Complex columns configurations in this work include simple column sequences, Petlyuk columns, columns with side-rectifiers and side strippers. It is assumed that condensers and reboilers are only placed at the extremes of the column sections. For modeling purposes, tray-by-tray models are considered and we neglect effects of mass transfer and assume that phase equilibrium is attained in every tray.

\section{Outline of Solution Strategy}

Tray-by-tray distillation synthesis models are very difficult to optimize due to the highly nonlinear and nonconvex equations that are involved, as well as to the large size of the corresponding formulations. Convergence problems are often found when solving these complex models (Dunnebier and Pantelides, 1999; Yeomans and Grossmann, 2000b; Bauer and Stichlmair, 1995). Therefore, there is a clear need for developing a computational strategy that exploits the nature of the decisions involved in this problem in order to yield robust and computationally effective models.

In the optimal synthesis of complex distillation configurations the discrete decision regarding the existence of each column has to be established. Moreover, if a given column exists in the configuration, it is also desired to optimize the feed tray location and the number of trays of that unit. Formulating and solving a single optimization problem to establish all the decisions simultaneously generally leads to a very difficult problem that often fails to converge. For these reasons, we first formulate the synthesis problem as a generalized disjunctive program, which does not have to be solved simultaneously and is amenable to decomposition. In particular, we propose an iterative decomposition strategy that exploits two major levels of decisions in the problem. In the first level, a configuration is derived by making the decision related to the selection of column sections. In this level each section is assigned a maximum number of trays in order to produce a bounding solution. In the second level, the feed tray location and the number of trays of the selected sections is optimized.

The proposed algorithm solves the disjunctive programming model by iteratively solving an MILP for selecting the sections, an MILP for selecting the trays of that configuration and an NLP subproblem for optimizing the particular design. A thermodynamic based NLP is solved for the initialization of this decom- 
position method. Several numerical examples are presented to illustrate the efficiency and robustness of the proposed method.

\section{Superstructure of Complex Configurations}

The construction of a general superstructure for complex distillation column systems is a nontrivial problem due to the large number of alternative designs that are possible. Different designs are obtained by making structural choices in the superstructure, such as the selection of the unit interconnections, feeds, products and heat exchange locations as well as the selection of trays in each column.

Most of the superstructures found in the literature are based on the superstructures proposed by Sargent and Gaminibandara for zeotropic and azeotropic cases (Sargent and Gaminibandara, 1976; Sargent, 1998). The superstructure proposed by these authors is represented in terms of the "State-Task-Network" (STN) introduced by Kondili et al. (1993). In Figure 1, the STN representation for a zeotropic mixture with components $A, B, C, D$ (decreasing volatility from $A$ to $D$ ) is shown. The circles represent possible states and the links between them are the tasks. The arrows indicate the direction of the net flows trough the network. From the feed stream, a distillate product with components, $A B C$, is obtained as well as a ternary bottom product, $B C D$. These products become the feed of subsequent units. Note that the states in the network are generated by removing the heaviest component from the distillate product and the lightest component from the bottom product. The tasks are defined as follows. All the states having the same components are joined, which is equivalent to coupling the units. In Figure 2, the column representation for the network presented in Figure 1 is shown. Note that the stripping section of column 2 and the rectifying section of column 3, both in the second level of Figure 2 (a), are coupled, which is equivalent to representing product, $B C$, in only one state. In all the levels of the Sargent-Gaminibandara superstructure the columns can be coupled and represented by one single unit (see Figure 2 (b)). For a mixture containing $N C$ components this representation will have $(N C-1)$ levels and a maximum of $\frac{1}{2} N C(N C-1)$ columns (Sargent and Gaminibandara, 1976).

The superstructure considered in this paper is based on the Reversible Distillation Sequence Model (RDSM) proposed by Fonyó (1974), which allows the introduction of thermodynamic aspects in the design (for details of the RDSM theory see Koehler et al., 1992; Barttfeld and Aguirre, 2003). This superstructure can be automatically generated for zeotropic as well as for azeotropic mixtures. In the latter case, a composition diagram of the mixture is assumed to be available.

The RDSM-based superstructure can be generated using the STN representation of Sargent (1998).

For the RDSM-based superstructure the states are defined in the same way as in the Sargent-Gaminibandara 
superstructure, but the tasks in this representation are different as seen in Figure 3 for a four component mixture. In order to approximate reversibility conditions, only products having the same composition can be represented in one state. As an example, the RDSM STN two different states are defined for the mixture, $B C$, in the representation of Figure 3. These states come from states, $A B C$, or $B C D$, and do not necessarily have the same composition. As a consequence of this fact, for separating a NC-zeotropic mixture, the RDSMbased superstructure has the same number of levels as the Sargent-Gaminibandara representation, but a larger number of columns, given by $2^{N C-1}-1$. The representation of the equipment for the RDSM-based superstructure for a four component zeotropic mixture is shown in Figure 4 (a). Note that in this representation columns 2 and 3 (second level) cannot be coupled. However, other representations are possible for the RDSM superstructure (see Koehler et al, 1992). In the RDSM representation considered column coupling is only possible in those columns that yield pure products, that is, in the last level of the superstructure. Note that columns 4 and 5 are integrated to produce product, $B$ as well as columns 6 and 7 to produce pure product $C$ (see Figure 4 (b)). Therefore, in the proposed superstructure it is not possible to represent in a level all the columns by one single unit as in the representation of Sargent and Gaminibandara of Figure 2. Only $2^{N C-3}$ columns integrations (single columns) can be found in the last level of the superstructure.

Each column in the superstructure of Figure 4 is represented by an adiabatic unit, and with one condenser and one reboiler. An index $k$ denotes each column in the superstructure. Each unit $k$ is represented by two sections, rect $k$ and $\operatorname{strip}_{k}$. The trays in each unit can be classified as intermediate or permanent trays (Yeomans and Grossmann, 2000b). Permanent trays perm $_{k}$ are those that are fixed in the superstructure. Each unit has three permanent trays: the feed tray and the top and bottom trays, $\operatorname{top}_{k}$ and $b_{k} t_{k}$, respectively, where the energy exchange takes place (see Figure 5). The representation selected for the column is the one that has been found to be the most effective to model distillation columns with GDP formulations (Barttfeld et al., 2003). Those trays that can disappear in the superstructure optimization are the intermediate trays $i t_{k}$. Note that the column sections contain intermediate trays and each section is located between two permanent trays. An upper bound on the number of trays $N T_{k}$ is assigned to each section of column $k$.

The columns in the superstructure are interconnected by feeds and products streams. The columns where multicomponent separations take place (columns 1, 2 and 3, Figure 4), are coupled by the feeds and products streams. Each column can be fed by primary and secondary feeds. The primary feed $F_{k, n}$ (see Figure 5) is the stream containing the components that are to be separated, while the secondary feeds $S_{2 k, n}$ and $S_{2 k+1, n}$ enter in the top and bottom trays providing part or the total amount of the reflux and reboil, respectively. The primary products $P V_{t o p_{k}}$ and $P L_{b o t_{k}}$ turn into the primary feed $F_{2 k, n}$ and $F_{2 k+1, n}$ of a pre- 
ceding column, respectively. Secondary products, $S_{k, n}$, turn into the secondary feeds of a previous unit, as seen in Figure 5.

As was mentioned above, the unit sections located in the last level of the scheme (columns 4-7 in Figure 4 (a)) where binary separations take place, can also be coupled if the same products emerge from them. In Figure 4, the stripping section of column 4 is integrated with the rectifying section of column 5. In previous work (Barttfeld and Aguirre, 2002), the integration of the sections in the RDSM-based was considered. However, in this work the general superstructure for the column integration is presented. In Figure 6, the general superstructure for the coupling of sections is shown. Note that the reflux of column $k+1$ can be provided either by the liquid product flow $P L_{b_{t}}$ from column $k$ bottom, by the condenser itself or by both. The same situation arises with the reboil stream in column $k$. Because these two sections are integrated, only one product $P P_{j}$ stream is obtained. The product can be formed with the contributions of the liquid bottom product $P L_{b o t_{k}}$ emerging column $k$ (note section $k+1$ may not exist), by the liquid top product $P L_{t o p_{k}}$ of column $k+1$ (note section $k$ may not exist) or by both. The total number of products streams is $3 * 2^{N C-1}$. The products that are rich in the most and the least volatile components are obtained not only in the columns of the last level of the superstructure. In Figure 4, the flow of the final product rich in component $A$ can be obtained by the liquid top product from column 4, in addition to the contributions of the liquid top products from column 1 and column 2.

The RDSM-based superstructure embeds conventional and complex columns configurations (Koehler et al, 1992). To illustrate this point, consider Figure 7 (a) where a general superstructure for a three components zeotropic mixture is shown. Also, consider that the stripping section of column 2 is not selected (see Figure 7 (b)). This then yields the side stripper configuration (see Figure 7 (c)) or the indirect sequence if there is no reverse flow from column 2 to column 1. Also these two configurations can arise if both sections of column 2 are not selected. Note that two equivalent structures exist for the side stripper configuration. The configuration shown in Figure 7 (c) is equivalent to the one in Figure 7 (d). In the same fashion, if the rectifying section of column 3 does not exist in the solution, either the side rectifier configuration or the direct sequence arise. As in the previous case, these two configurations are also obtained if column 3 is not selected.

If all the sections are selected in the superstructure of Figure 7, except for the rectifying section of column 2 , the design is not a feasible configuration. The same situation occurs if all the sections are selected 
except for the stripping section of column 3. To avoid these infeasible designs, logic constraints will be included in the formulation presented in the next section.

The superstructure considered in this work can also be extended for azeotropic distillation. Due to the existence of distillation boundaries in this case, the order of the relative volatility of the components cannot be predefined. As a consequence, it is not possible to define the states a priori as in the zeotropic case. A composition diagram showing the distillation boundaries is needed to define the feasible states that can be achieved from a given feed. Consider a ternary azeotropic mixture with a topology composition diagram given by Figure 8 (a). This mixture has one azeotrope between the middle and the heaviest component. In order to generate the STN representation, the mass balances for each reversible separation task (Koehler et al., 1992; Fonyó, 1974; Barttfeld and Aguirre, 2002; Barttfeld and Aguirre, 2003) have to be defined in this diagram. In Figure 8 (a), the feed composition is denoted by $F$. Two products are obtained from this feed: a distillate product $D 1$ and a bottom product $B 1$. Note that due to the existence of a distillation boundary, product $D 1$ is a ternary mixture instead of binary, like in the zeotropic case. Assuming that $D 1$ is located in a different distillation region than the feed ( $D 1$ crosses the distillation boundary), the procedure for generating the states follows in the same fashion as in the zeotropic case. However, for azeotropic mixtures, the azeotrope is considered as a pseudo components (Sargent, 1998). The complete states and tasks definitions are presented in the STN representation in Figure 8 (b). The superstructure for the separation of this mixture into pure products is derived in Figure 8 (c). Note that the stream with the azeotrope composition is recycled to be fed in the first column of the scheme.

\section{GDP Formulation}

In this section, the Generalized Disjunctive Programming (Turkay and Grossmann, 1996) formulation for the optimal synthesis for the separation of a $N C$-component zeotropic mixture is presented. Let $F o$ and $z f o_{i}$ be the feed flow rate and composition, respectively. Let $C$ be the set of components $i$ present in the feed: $C=\{i \mid i=1, \ldots, N C\}$. As defined in the previous section, let the index $k$ denote each column in the sequence: $N C O L=\left\{k \mid k=1, \ldots, K=2^{N C-1}-1\right\}$. Let NTRAY be the set of potential trays NTRAY $=\{n \mid n=1, \ldots, N T\}$ and let $N_{k}$ represents the subset of trays $n_{k}$ in column $k$ : $N_{k}=\left\{n_{k} \mid n_{k}=1, \ldots, N T_{k}\right\}$. Also consider the general RDSM superstructure shown in Figure 9 for the separation of a $N C$-zeotropic mixture. 
The formulation of the discrete choices regarding the structural optimization are modeled through embedded disjunctions. In a first level of decision, boolean variables $Y_{s}$ are defined to denote the existence of a section $s$. If a value of true is assigned to the variable $Y_{s}$, the section $s$ is selected for which $n t r a y_{s}$ trays are to be selected in this section. If the section is not selected, the internal vapor and liquid flows are set to zero and no trays are activated in this section.

In a second level of decision, boolean variables $W_{n}$ are assigned to every potential tray of an active section to denote its existence. If a value of true is assigned to $W_{n}$, the tray $n$ is selected and the vapor-liquid equilibrium equations are applied. Otherwise, if the tray is not selected, the internal vapor and liquid streams are bypassed and no mass transfer process takes place. The embedded disjunction for a given section $s$ is formulated as follows, where each term of the disjunction applies for components $i \in C$ :

$$
\left[\begin{array}{c}
\operatorname{Ytray}_{s}=\sum_{n \in \sec _{s}} \operatorname{stg}_{n} \\
{\left[\begin{array}{l}
W_{n} \\
f_{n, i}^{L}=f\left(T_{n}^{L}, P_{n}, x_{n, i}\right) \\
f_{n, i}^{V}=f\left(T_{n}^{V}, P_{n}, y_{n, i}\right) \\
f_{n, i}^{L}=f_{n, i}^{V} \\
T_{n}^{V}=T_{n}^{L} \\
L I Q_{n, i}=L_{n} x_{n, i} \\
V A P_{n, i}=V_{n} y_{n, i} \\
s t g_{n}=1
\end{array}\right] \vee\left[\begin{array}{c}
\neg W_{n} \\
f_{n, i}^{L}=0 \\
f_{n, i}^{V}=0 \\
T_{n}^{V}=T_{n+1}^{V} \\
T_{n}^{L}=T_{n-1}^{L} \\
V_{n}=V_{n+1} \\
L_{n}=L_{n+1} \\
x_{n, i}=x_{n-1, i} \\
y_{n, i}=y_{n+1, i} \\
s t g_{n}=0
\end{array}\right] \forall n \in \sec _{s}} \\
f_{n, i}^{V}=0 \\
f_{n, i}^{V}=0 \\
T_{n}^{V}=T_{n+1}^{V} \\
T_{n}^{L}=T_{n-1}^{L} \\
V_{n}=0 \\
L_{n}=0 \\
x_{n, i}=x_{n-1, i} \\
y_{n, i}=y_{n+1, i} \\
n t r a y_{s}=0
\end{array}\right] \forall \in S
$$

In (1) $S$ is the set of rectifying and stripping column sections in the sequence: $S=\left\{s \mid s=\right.$ rect $_{1}$, strip $_{1}$, rect $_{2}$, strip $_{2}, \ldots$, rect $_{K}$, strip $\left._{K}\right\}$. In order to assign a set of trays to a section, the set $\sec _{s}$ is defined to denote the set of potential intermediate trays that belong to section $s$.

The design specifications and logic propositions are given by the general expressions (2) and (3), which involve only boolean variables:

$\Omega\left(Y_{s}\right)=$ True $\forall s \in S$ 
In equation (3), the set IT denotes all the intermediate trays of the superstructure: $I T=\bigcup_{k=1}^{K} i t_{k}$. These propositions model feasibility conditions for the separation and logic expressions. Feasibility conditions can be formulated for zeotropic mixtures considering that the minimum number of column sections required to achieve pure products is 2(n-1) (Agrawal, 1996). To illustrate feasibility conditions, consider a zeotropic ternary mixture. According to the superstructure shown in Figure 7 (a), the following logic feasibility propositions apply:

$$
\begin{aligned}
& \neg Y_{\text {strip }_{2}} \Rightarrow Y_{\text {strip }_{3}} \wedge Y_{\text {rect }_{3}} \\
& \neg Y_{\text {rect }_{3}} \Rightarrow Y_{\text {strip }_{2}} \wedge Y_{\text {rect }_{2}} \\
& \neg Y_{\text {rect }_{2}} \Rightarrow \neg Y_{\text {strip }_{2}} \\
& \neg Y_{\text {strip }_{3}} \Rightarrow \neg Y_{\text {rect }_{3}}
\end{aligned}
$$

Equation (4) imposes the condition that if the stripping section of column 2 is not selected ( $Y_{\text {strip2 }}$ is false), column 3 has to be selected ( $Y_{\text {strip } 3}$ and $Y_{\text {strip } 3}$ true) in order to achieve a feasible design. The analogous situation is model in equation (5) if the rectifying section of column 3 is not selected. Equation (6) avoids that the stripping section of column 2 is selected if the rectifying section of this column was previously activated. For column 3, equation (7) avoids the selection of the rectifying section if the stripping section of this column was not previously activated.

For the trays which belong to selected sections, logic expressions are considered to avoid the possibility of obtaining multiple solutions with the same objective function value. Equations (8) and (9) enforce that the selected trays be selected above and below the feed tray (Yeomans and Grossmann, 2000b):

$$
\begin{aligned}
& {\left[\begin{array}{c}
Y_{s} \\
W_{n+1} \Rightarrow W_{n}
\end{array}\right] \vee\left[\begin{array}{c}
\neg Y_{s} \\
W_{n}=\text { false }
\end{array}\right] \forall s \in \text { RECT, } n \in \text { sec }_{s}} \\
& {\left[\begin{array}{c}
Y_{s} \\
W_{n} \Rightarrow W_{n+1}
\end{array}\right] \vee\left[\begin{array}{c}
\neg Y_{s} \\
W_{n}=\text { false }
\end{array}\right] \forall s \in \text { STRIP }, n \in \text { sec }_{s}}
\end{aligned}
$$

Note that the set $R E C T$ contains all the rectifying sections: $R E C T=\left\{\right.$ rect $_{1}$, rect $\left._{2}, \ldots, r e c t_{K}\right\}$ and the set $S T R I P$ all the stripping sections: $S T R I P=\left\{\right.$ strip $_{1}$, strip $_{2}, \ldots$, strip $\left._{K}\right\}$. Also, let $N F$ be the set of all feed streams in the superstructure: $N F=\{n f \mid n f=F, S, L\}$. 
The VLE and equilibrium conditions are applied for all the permanent trays of the sequence in equations (10). The set of permanent stages in each column $k$ are defined as follows: $\operatorname{perm}_{k}=\operatorname{prod}_{k} \bigcup_{n f}$ feed $_{n f, k}$, where $\operatorname{prod}_{k}$ is a tray where a product is withdrawn and feed $d_{n f, k}$ is a tray where feed $n f$ enters in column $k$. Then, the set of all permanent trays in the superstructure is given by PERM $=\bigcup_{k=1}^{K} \operatorname{perm}_{k}$. Equations (11) include the summation of the mole fractions and the definition of the enthalpies for the internal vapor and liquid streams:

$$
\begin{aligned}
& f_{n, i}^{L}=f\left(T_{n}, P_{n}, x_{n, i}\right) \\
& f_{n, i}^{V}=f\left(T_{n}, P_{n}, y_{n, i}\right) \\
& f_{n, i}^{V}=f_{n, i}^{L} \\
& T_{n}^{V}=T_{n}^{L} \quad \forall n \in P E R M, i \in C \\
& L I Q_{n, i}=L_{n} x_{n, i} \\
& V A P_{n, i}=V_{n} y_{n, i} \\
& \operatorname{stg}_{n}=1 \\
& \sum_{i=1}^{N C} x_{n, i}=1 \\
& \sum_{i=1}^{N C} y_{n, i}=1 \\
& \forall n \in N T R A Y \\
& h l i q_{n}=f\left(T_{n}^{L}, L I Q_{n, i}\right) \\
& \operatorname{hvap}_{n}=f\left(T_{n}^{V}, V A P_{n, i}\right)
\end{aligned}
$$

Equations (12) define the individual feed flow for each feed stream $n f$.

$$
F E E D_{n f, n, i}=F_{n f, n} z f_{n f, i} \quad \forall n \in P E R M, n \in \text { feed }_{n f, k}, n f \in N F, i \in C
$$

The liquid and vapor intermediate product individual flows are defined in equation (13) as well as the liquid and vapor products enthalpies. In this constraint, $N P R O D$ is the set of products of the superstructure: $N P R O D=\bigcup_{k=1}^{K} \operatorname{nprod}_{k}$, where $\operatorname{nprod}_{k}$ is the set of products stages in each column $k$ : $\operatorname{nprod}_{k}=\operatorname{top}_{k} \cup \operatorname{bot}_{k} \bigcup_{n p} \operatorname{sidep}_{n p, k}$. Note that the set sidep $n p, k$ refers to the candidate trays from where a product $n p$ can be withdrawn in column $k$. 


$$
\left.\begin{array}{l}
\operatorname{PLIQ}_{n, i}=P L_{n} x_{n, i} \\
\operatorname{PVAP}_{n, i}=P V_{n} y_{n, i} \\
\text { hpliq }_{n}=f\left(T_{n}^{L}, P L I Q_{n, i}\right) \\
\text { hpvap }_{n}=f\left(T_{n}^{V}, P V A P_{n, i}\right)
\end{array}\right\} \forall n \in P E R M, n \in N P R O D, i \in C
$$

Mass and energy balances are formulated for every tray. Equations (14) model the feed trays, equations (15) are applied to all intermediate trays, and equations (16) and (17) model top and bottom trays, respectively. Note that $T O P=\bigcup_{k=1}^{K} t_{0}$ and $B O T=\bigcup_{k=1}^{K} b o t_{k}$. Equations (18) are applied to those trays from where side products emerge.

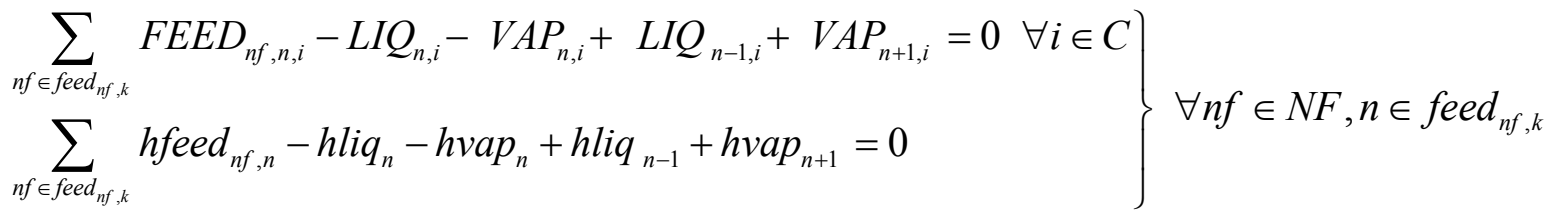

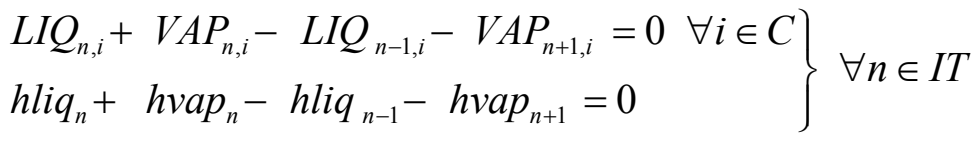

$$
\begin{aligned}
& \left.\begin{array}{l}
V A P_{n+1, i}-\operatorname{LIQ}_{n, i}-\operatorname{PLIQ}_{n, i}-\operatorname{PVAP}_{n, i}=0 \forall i \in C \\
\operatorname{hvap}_{n+1}-\text { hliq }_{n}-\text { hpliq }_{n}-\text { hpvap }_{n}-Q_{n}=0
\end{array}\right\} \forall n \in T O P \\
& \left.\begin{array}{l}
L I Q_{n-1, i}-\operatorname{VAP}_{n, i}-\operatorname{PLIQ}_{n, i}=0 \quad \forall i \in C \\
\text { hliq }_{n-1}-\text { hvap }_{n}-\operatorname{hpliq}_{n}+Q_{n}=0
\end{array}\right\} \forall n \in B O T
\end{aligned}
$$

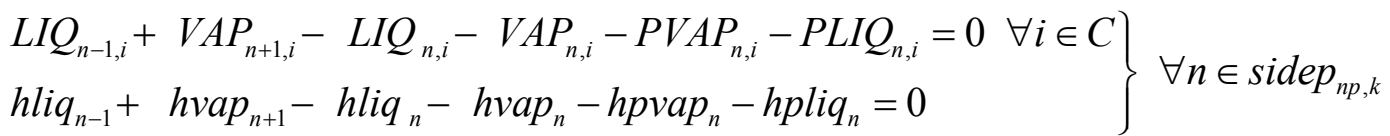

The energy requirements in the condenser $Q c_{k}$ and reboiler $Q h_{k}$ of every column $k$ are defined in equations (19):

$$
\left.\begin{array}{l}
Q c_{k}=\sum_{n \in \text { top }_{k}} Q_{n} \\
Q h_{k}=\sum_{n \in \text { bot }_{k}} Q_{n}
\end{array}\right\} \forall k \in N C O L
$$

Total mass and energy balances are formulated for the superstructure in equations (20). In constraints (21) mass and energy balances are formulated for each column $k$ of the superstructure (see Figure 9). 


$$
\begin{aligned}
& \sum_{n \in \text { feed }_{F_{1}, 1}} F_{F_{1}, n}=\sum_{j=1}^{J} P P_{j} \\
& \sum_{n \in \text { feed }_{F_{1}, 1}} F E E D_{F_{1}, n, i}=\sum_{j=1}^{J} P z_{j, i} \quad \forall i \in C \\
& \sum_{n \in \text { feed }_{F, 1}} h_{\text {feed }} \text { F1,n }_{1}+\sum_{k=1}^{K}\left(Q h_{k}-Q c_{k}\right)=\sum_{j=1}^{J} P h_{j} \\
& \left.\begin{array}{l}
\sum_{n \in N_{k}} \sum_{n f \in \text { feed }_{n f, k}} F_{n E E D_{n f, n, i}=\sum_{n \in N_{k}}\left(P L I Q_{n, i}+P^{\prime} V A P_{n, i}\right) \quad \forall i \in C} \\
\sum_{n \in N_{k}} \sum_{n f \in \text { feed }_{n f, k}} \text { hfeed }_{n f, n}+Q h_{k}-Q c_{k}=\sum_{n \in N_{k}}\left(\text { hpliq }_{n}+\text { hpvap }_{n}\right)
\end{array}\right\} \forall k \in N C O L
\end{aligned}
$$

Column interconnection balances are considered next. The problem feed flow, composition and enthalpy are defined in equations (22):

$$
\begin{aligned}
& \sum_{n \in \text { feed }_{F 1,1}} F_{F 1, n}=F O \\
& \sum_{n \in \text { feed }_{F 1,1}} F E E D_{F 1, n, i}=F o z f o_{i} \quad \forall i \in C \\
& \sum_{n \in \text { feed }_{F 1,1}} h_{F e e d}
\end{aligned}
$$

Primary feeds are defined by equations (23). The vapor (liquid) product emerging from the top (bottom) of a column becomes the feed of a subsequent column of the sequence (see Figure 9).

$$
\begin{aligned}
& P V_{\text {top }_{k}}=\sum_{n \in \text { feed }_{F_{2 k}, k}} F_{F_{2 k}, n} \\
& \text { PVAP }_{\text {top }_{k}, i}=\sum_{n \in \text { feed }_{F_{2 k}, k}} \text { FEED }_{F_{2 k}, n, i} \quad \forall i \in C \\
& \text { hpvap }_{\text {top }_{k}}=\sum_{n \in \text { feed }_{F_{2 k}, k}} \text { hfeed }_{F_{2 k}, n} \\
& P L_{\text {bot }_{k}}=\sum_{n \in \text { feed }_{F_{2 k+1, k}}} F_{F_{2 k+1}, n} \\
& P L I Q_{\text {bot }_{k}, i}=\sum_{n \in \text { feed }_{F_{2 k+1, k}}}{ }{ }^{2} E E D_{F_{2 k+1}, n, i} \quad \forall i \in C \\
& \text { hpliq }_{\text {bot }_{j}}=\sum_{n \in \text { feed }_{F_{2 k+1, k}}} \text { hfeed }_{F_{2 j+1}, n} \\
& \forall k \in N C O L, 1 \leq k \leq K-1
\end{aligned}
$$

The secondary feeds are defined in equations (24) and (25). The liquid (vapor) side product emerging from a column becomes the secondary feed of a preceding column in the superstructure. 


$$
\left.\begin{array}{l}
\sum_{n \in \text { sidep p }_{S_{k}, k}} P L_{n}=\sum_{n \in \text { feed }_{S_{k}, k}} F_{S_{k}, n} \\
\sum_{n \in \text { sidep }_{S_{k}, k}} P L I Q_{n, i}=\sum_{n \in \text { feed }_{S_{k}, k}} \text { FEED }_{S_{k}, n, i} \quad \forall i \in C \\
\sum_{n \in \text { sidep }_{S_{k}, k}} \text { hpliq }_{n}=\sum_{n \in \text { feed }_{S_{k}, k}} \text { hfeed }_{S_{j}, n}
\end{array}\right\} \forall k \in N C O L, 2 \leq k \leq K, k \text { even }
$$

Note that the liquid side product from an even column becomes the secondary feed entering in the rectifying section of a preceding column (i.e. in Figure 9, the product $S_{2}$ is the secondary feed entering column 1 top).

$$
\left.\begin{array}{l}
\sum_{n \in \text { sidep }_{S_{k}, k}} P V_{n}=\sum_{n \in \text { feed }_{S_{k}, k}} F_{S_{k}, n} \\
\sum_{n \in \text { sidep }_{S_{k}, k}} P V A P_{n, i}=\sum_{n \in \text { feed }_{s_{k}, k}} \text { FEED }_{S_{k}, n, i} \quad \forall i \in C \\
\sum_{n \in \text { sidep }_{S_{k}, k}} \text { hpvap }_{n}=\sum_{n \in \text { feed }_{S_{k}, k}} \text { hfeed }_{S_{j}, n}
\end{array}\right\} \forall k \in N C O L, 3 \leq k \leq K, k \text { odd }
$$

In equation (25), the side vapor product exiting from an odd column is the secondary feed entering in the stripping section of a preceding column (i.e. in Figure 9, the product $S_{3}$ is the secondary feed entering column 1 bottom). The balances for those sections which are integrated are modeled from equations (26) to (31) (see Figure 6). Equations (26) define the secondary feeds $F_{L_{k}, n}$.

$$
\begin{aligned}
& \begin{array}{l}
P L_{b o t_{k}} \geq \sum_{n \in \text { feed }_{L_{k}, k}} F_{L_{k}, n} \\
P L I Q_{b o t_{k}, i} \geq \sum_{n \in \text { feed }_{L_{k}, k}} F E E D_{L_{k}, n, i} \quad \forall i \in C
\end{array} \\
& \text { hpliq }_{\text {bot }_{k}} \geq \sum_{n \in \text { feed }_{L_{k}, k}} \text { hfeed }_{L_{k}, n} \\
& P V_{\text {top }_{k+1}}=\sum_{n \in \text { feed }_{L_{k+1}, k}} F_{L_{k+1}, n} \\
& P V A P_{\text {top }_{k+1}, i}=\sum_{n \in \text { feed }_{L_{k+1}, k}}{ } E E D_{L_{k+1}, n, i} \quad \forall i \in C \\
& \text { hpvap }_{\text {top }_{k+1}}=\sum_{n \in \text { feed }_{L_{k+1}, k}} \text { hfeed }_{L_{k+1}, n} \\
& \forall k \in N C O L, k \in\left\{2^{N C-1}-1-N C+m\right\}, 0 \leq m \leq K-1, m \text { even }
\end{aligned}
$$

Equations (27) define the products $P P_{j}$ emerging from totally integrated sections. The index $j$ denotes each final product $F P=\left\{j \mid j=1, \ldots, J=3^{*} 2^{N C-1}\right\}$. 


$$
\begin{aligned}
& P L_{b o t_{k}}-\sum_{n \in \text { feed }_{L_{k}, k}} F_{L_{k}, n}+P L_{t o p_{k+1}}=P P_{j} \\
& \left.P L I Q_{\text {bot }_{k}, i}-\sum_{n \in \text { feed }_{L_{k}, k}} \text { FEED }_{L_{k}, n, i}+P L I Q_{\text {too }_{k+1}, i}=P z_{j, i} \quad \forall i \in C\right\} \\
& \text { hpliq }_{\text {bot }_{k}}-\sum_{n \in \text { feed }_{L_{k}, k}} \text { hfeed }_{L_{k}, n}+\text { hpliq }_{\text {top }_{k+1}}=P h_{j} \\
& \forall k \in N C O L, j \in F P, k \in\left\{2^{N C-1}-1-N C+m\right\} \text {, } \\
& j=3 m-1,0 \leq m \leq K-1, m \text { even }
\end{aligned}
$$

Constraints (28) define the final product that is rich in the lightest component $P P_{1}$ :

$$
\left.\begin{array}{l}
P P_{j}=P L_{t o p_{1}}+\sum_{k \in K z_{1}} P L_{t o p_{k}} \\
P z_{j, i}=P L I Q_{t o p_{1}, i}+\sum_{k \in K z_{1}} P L I Q_{\text {top }_{k}, i} \quad \forall i \in C \\
P h_{j}=h p l i q_{\text {top }_{K-N C}}+\sum_{k \in K z_{1}} h \operatorname{liq}_{\text {top }_{k}}
\end{array}\right\} \forall j \in F P, j=1
$$

In equations (28), Kz $z_{1}$ is the subset of NCOL defined as follows: $K z_{1}=\left\{k \mid 2 \leq k \leq 2^{N C-2}, k \in\left\{\sum_{r=2}^{m} 2^{r-1}\right\}\right.$ with $\left.1 \leq m \leq N C\right\}$. Note that the product rich in the lightest component $P P_{1}$, is formed with the top product of column 1 and with the contributions of all the top products of the first columns of each level (see Figure 9). Then, $K z_{1}$ is the subset defining the first column at each level of the superstructure.

Constraints (29) define the final product that is rich in the heaviest component $P P_{J}$ :

$$
\left.\begin{array}{l}
P P_{j}=P L_{\text {bot }_{K}}+\sum_{k \in K z_{2}}\left(P L_{\text {bot }_{k}}-\sum_{n \in \text { feed }_{F_{2} k+1, k}} F_{F_{2 k+1}, n}\right) \\
P z_{j, i}=P L I Q_{b o t_{K}, i}+\sum_{k \in K z_{2}}\left(P L I Q_{\text {bot }_{k}, i}-\sum_{n \in \text { feed }_{F_{2 k+1}, k}} F E E D_{F_{2 k+1}, n, i}\right) \quad \forall i \in C \\
P h_{j}=\text { hpliq }_{\text {bot }_{K}}+\sum_{k \in K z_{2}}\left(\text { hpliq }_{\text {bot }_{k}}-\sum_{n \in \text { feed }_{F_{2} k+1, k}} h_{f e e d} F_{F_{2 k+1}, n}\right)
\end{array}\right\} \forall j \in F P, j=J
$$

In equations (29), Kz $z_{2}$ is the subset of NCOL defined as follows: $K z_{2}=\left\{k_{r} \mid 1 \leq k_{r} \leq K, k_{r}=2 k_{r-1}+1, k_{0}=1, r \in \mathbb{Z}\right\}$. Note that the product that is rich in the heaviest component $P P_{J}$, is formed with the bottom product of column $K$ and with the contributions of all the bottom prod- 
ucts of the last columns of each level (see Figure 9). Then, $K z_{2}$ is the subset defining the last column at each level of the superstructure.

Next, the final products emerging from the nonintegrated rectifying section are defined. Consider Figure 10, where the superstructure is shown for a five component feed. Two types of top products can be defined. Product $\mathrm{PP}_{4}$ (see Figure 10) is formed by the contributions of the top products of column 10 and column 5. If column 10 is not selected, product $P P_{4}$ rich in component $B$ will emerge directly from column 5. For a multicomponent mixture, equations (30) model this product as follows:

$$
\begin{aligned}
& \left.\begin{array}{l}
P P_{j}=P L_{t o p_{k}}+P L_{t o p_{k / 2}} \\
P z_{j, i}=P L I Q_{t o p_{k}, i}+P L I Q_{t o p_{k / 2}, i} \\
P h_{j}=h p l i q_{t o p_{k}}+h p l i q_{t o p_{k / 2}}
\end{array}\right\} \\
& k \in N C O L, j \in F P, k \in\left\{2^{N C-2}+2+m\right\} \text {, } \\
& 5 \leq k<K, j=4,10,16, \ldots, m=0,4,8 \ldots
\end{aligned}
$$

Note that in Figure 10, product $P P_{7}$ is formed by the contributions of the top product of column 12 as well as by the top products of columns 3 and 6 . If column 12 is not selected in the superstructure, then product $P P_{7}$ will emerge from the top of column 6. If both columns 6 and 12 are not selected, this product will exit from the top section of column 3. For a multicomponent feed the definition of this type of final product is given by equations (31):

$$
\begin{gathered}
P P_{j}=P L_{t o p_{k}}+\sum_{K z_{3}} P L_{t o p_{k}} \\
\left.P z_{j, i}=P L I Q_{t o p_{k}, i}+\sum_{K z_{3}} P L I Q_{t o p_{k}, i} \quad \forall i \in C\right\} \\
P h_{j}=h \text { pliq }_{t o p_{k}}+\sum_{K z_{3}} h p l i q_{t o p_{k}} \\
k \in N C O L, j \in F P, k \in\left\{2^{N C-2}+4+m\right\}, \\
12 \leq k<K, j=7,14, \ldots, m=0,4,8 \ldots
\end{gathered}
$$

In the above equations (31), Kz 3 is the subset of NCOL defined as follows: $K z_{3}=\left\{k_{r} \mid 3 \leq k_{r} \leq K, k_{r}=2 k_{r-1}, k_{0}=3, r \in \mathbb{Z}\right\}$. Note that for a five component feed, $K z_{3}$ is the subset containing columns 3 and 6 .

The products from non integrated stripping sections are defined next. 


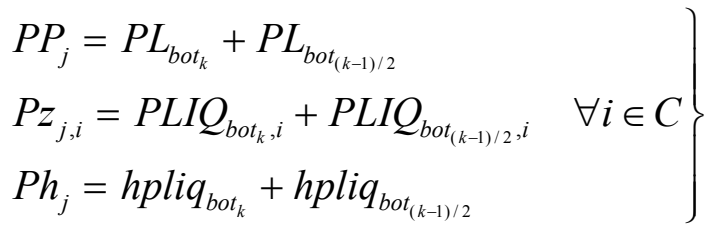

$$
\begin{aligned}
& k \in N C O L, j \in F P, k \in\left\{2^{N C-2}+1+m\right\}, \\
& k<K, j=3,9, \ldots, m=0,4,8 \ldots \\
& P P_{j}=P L_{\text {bot }_{k k}}+\sum_{k \in K z_{4}}\left(P L_{\text {bot }_{k}}-\sum_{n \in \text { feed }_{F_{2 k+1, k}}} F_{F_{2 k+1}, n}\right) \\
& \left.P z_{j, i}=P L I Q_{b o t_{k l}, i}+\sum_{k \in K_{4}}\left(P L I Q_{\text {bot }_{k}, i}-\sum_{n \in \text { feed }_{F_{2 k+1}, k}} F E E D_{F_{2 k+1}, n, i}\right) \quad \forall i \in C\right\} \\
& P h_{j}=\text { hpliq }_{\text {bot }_{k k}}+\sum_{k \in K_{z_{4}}}\left(\text { hpliq }_{\text {bot }_{k}}-\sum_{n \in \text { feed }_{F_{2 k+1, k}}} \text { hfeed }_{F_{2 k+1}, n}\right) \\
& k k \in N C O L, j \in F P, k \in\left\{2^{N C-2}+3+m\right\}, \\
& k k<K, j=6,12, \ldots, m=0,4,8 \ldots
\end{aligned}
$$

Equation (32) models the final products which are obtained by the contribution of a column of the last level of the superstructure and by the bottom product emerging from a previous unit. Note that in Figure 10, the bottom products of column 4 and 9 contribute to the product $P P_{3}$. In case column 9 is not selected, the product $P P_{3}$ will emerge from the stripping section of column 4 .

In equations (33), $\mathrm{Kz}_{4}$ is the subset of NCOL defined as follows: $K z_{4}=\left\{k_{r} \mid 2 \leq k_{r} \leq k k, k_{r}=2 k_{r-1}+1, k_{0}=2, r \in \mathbb{Z}\right\}$. Note that $K z_{4}$ defines the columns which contributes with the final product. In Figure 10, equation (33) would model product $P P_{6}$. For this example, $K z_{4}$ is the set containing column 2 and column 5 . Also note that the set $k$ was renamed as $k k$ because a reference point is needed to define $\mathrm{Kz}_{4}$.

The column diameters are computed in equation (34). However, a uniform diameter is considered for every column $k$ in (35) as well as the actual number of trays in each column.

$$
\begin{array}{ll}
\operatorname{Dcol}_{n}=f\left(T_{n}^{V}, P_{n}, V_{n}\right) \quad \forall n \in N T R A Y \\
D c_{s} \geq \text { Dcol }_{n} & \forall n \in \sec _{s} \\
N T_{k}=\sum_{n \in N_{k}} \operatorname{stg}_{n} & \forall k \in N C O L
\end{array}
$$

The individual flows and enthalpies of the final products are defined in equations (36). Constraints (37) define the specified recoveries $\varepsilon_{i}$ and purities $\tau_{i}$ : 


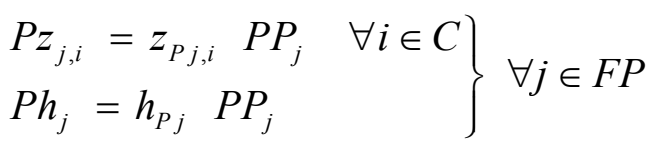

$$
\begin{aligned}
& \left.\begin{array}{l}
P z_{j, i} \geq \varepsilon_{i} \text { Fo } \quad z f o_{i} \\
z P_{j, i} \geq \tau_{i}
\end{array}\right\} \forall j \in F P, j=i, i \in C
\end{aligned}
$$

Since the problem consists of designing a distillation sequence involving minimum cost, the economic objective function in (38) involving the total annualized cost (TAC) of equipment and utilities is minimized subject to the constraints (1)-(3), (8)-(37), (39).

$\min T A C=C i n v+C o p$

Cop $=\sum_{k=1}^{K}\left(m w_{k}\right.$ Ccool $+m v_{k}$ Csteam $)$

\section{Solution Procedure}

In order to solve the model presented in section 5, an algorithmic procedure will be proposed. The embedded disjunction given by equation (1) models the discrete decisions related to the sequence structure and number of trays. Due to the difficulty in solving the entire problem, the GDP model proposed in section 4 will be decomposed into two levels of decisions within an iterative procedure. In a first level, the column sections are selected while in the second level the optimization of the number of trays is performed for the sections selected in levels.

Due to the size, nonlinearities and nonconvexities that are involved in each of the subproblems, good initial values and bounds have to be provided in order to achieve the convergence of the model. For that reason, a preprocessing phase is considered in the solution of the economic optimization problem. The main objective of this phase is to generate a good initial solution for the optimization of the sequence. The preprocessing phase for zeotropic mixtures was modeled and developed in a previous work where detailed explanations can be found (Barttfeld y Aguirre, 2003). In this work, the preprocessing procedure is generalized and extended for a ternary azeotropic case (see Appendix A). The optimization problems involved in each of the two levels are described next.

\subsection{Section Optimization GDP Problem}

This tray-by-tray model considers each column section with the number of trays fixed at the maximum value $\gamma$ so that if the section is selected, all the trays within the section are active but with a lower bound 
for the cost (i.e. minimum number of trays). The idea is to provide a lower bound to the cost by providing the maximum scope for separation while underestimating the investment cost. The embedded disjunction given by equation (1) reduces then into the following simple disjunction:

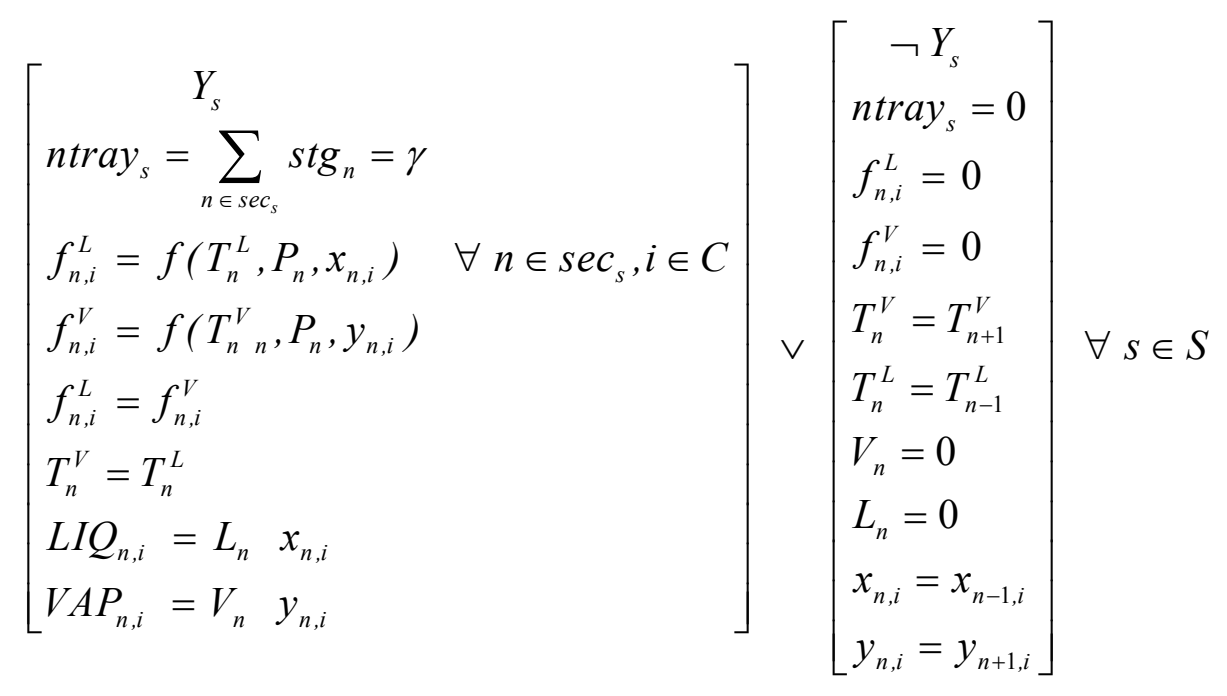

If $Y_{S}$ is true the section $s$ is selected and the upper bound $\gamma$ of the number of trays become active. Then, the VLE equations are applied to these trays. If the section is not selected, the VLE are not imposed and the vapor and liquid internal flows are set to zero.

The constraints for this problem are given by equations (2), (3), (10)-(31) and equations (36)-(39) and equation (40). The problem then consists in minimizing the total annualized cost $T A C$ which is defined in equation (38). In addition, the following equations are added to the model. The investment cost Cinv involves the cost for the column trays $C_{t r a y}$, the cost of the column shell $\mathrm{Ccol}_{S}$ and the condenser and reboiler costs Ccond $_{k}$ and $\mathrm{Creb}_{k}$, respectively (see equation (41)). Equation (42) are global constraints which define the trays and column shell costs.

$$
\begin{aligned}
& C i n v=\sum_{s}\left(\text { Ctray }_{s}+\text { Ccol }_{s}\right)+\sum_{k=1}^{K}\left(\text { Ccond }_{k}+\text { Creb }_{k}\right) \\
& \left.\begin{array}{l}
\operatorname{Ctray}_{s}=\gamma^{l o} \phi_{1} \\
\operatorname{Ccol}_{s}=\gamma^{l o} \phi_{2}
\end{array}\right\} \forall s \in S
\end{aligned}
$$

If a section $s$ is selected, a number of $n$ tray ${ }_{s}$ is assigned to each column section. However, to underestimate the investment costs, a lower bound for the number of trays, $\gamma^{l o}$, and the parameters $f_{1}$ and $f_{2}$ are considered in equation (42). This model does not include the calculation of the columns diameters. A constant average diameter is considered for each unit and defined as $f_{1}=f_{t}\left(D c_{k}\right)$ and $f_{2}=f_{c}\left(D c_{k}\right)$. The value for the 
diameter used can be easily computed from the tray-by-tray preprocessing phase model by adding to the constraints of this problem equations (34) and (35).

In summary, by solving the section optimization problem a candidate configuration with a lower bound for the cost is obtained. In the context of the algorithm the MILP approximation to the GDP problem will be used.

\subsection{Tray Optimization GDP Problem}

This optimization problem is formulated in a reduced space since the optimization is carried out over a candidate configuration instead over the initial superstructure. This problem is solved in a reduced space since the sections that are not selected are excluded from this problem.

The embedded disjunction which models the discrete decision of selecting the number of trays of active sections is given by the following constraint which is only applied to those trays within selected sections $\left(Y_{S}=\right.$ True):

$$
\left[\begin{array}{c}
W_{n} \\
f_{n, i}^{L}=f\left(T_{n}^{L}, P_{n}, x_{n, i}\right) \\
f_{n, i}^{V}=f\left(T_{n}^{V}, P_{n}, y_{n, i}\right) \\
f_{n, i}^{L}=f_{n, i}^{V} \\
T_{n}^{V}=T_{n}^{L} \\
\operatorname{LI}_{n, i}=L_{n} x_{n, i} \\
V A P_{n, i}=V_{n} y_{n, i} \\
\operatorname{stg}_{n}=1
\end{array}\right] \vee\left[\begin{array}{c}
\neg W_{n} \\
f_{n, i}^{L}=0 \\
f_{n, i}^{V}=0 \\
T_{n}^{V}=T_{n+1}^{V} \\
T_{n}^{L}=T_{n-1}^{L} \\
V_{n}=V_{n+1} \\
L_{n}=L_{n+1} \\
x_{n, i}=x_{n-1, i} \\
y_{n, i}=y_{n+1, i} \\
s t g_{n}=0
\end{array}\right] \forall n \in \sec _{s}, \text { for } Y_{s}=\text { True }
$$

If $W_{n}$ is assigned a value of true, the tray $n$ is selected and the VLE equations are applied. Otherwise, the vapor and liquid streams are by passed and no mass transfer process takes place.

The other constraints of this problem are given by equations (3), (8), (9), (10) to (31), and equations (34) to (39). Also, equation (44) is included for the computation of the investment cost as well as equations (45) which model the cost for the trays and the column shell.

$$
\operatorname{Cinv}=\sum_{k=1}^{K}\left(\text { Ccond }_{k}+\text { Creb }_{k}+\text { Ctray }_{k}+\text { Ccol }_{k}\right)
$$


$\left.\begin{array}{l}\operatorname{Ctray}_{k}=N T_{k} f_{t}\left(D c_{s}\right) \\ \operatorname{Ccol}_{k}=N T_{k} f_{c}\left(D c_{s}\right)\end{array}\right\} \forall k \in N C O L, s \in S$

These equations are global constraints that are functions of the number of trays of each unit $N T_{k}$ and also of the columns diameter, which is modeled in equations (34) and (35). The MILP approximation to this problem is also used in the algorithm in the next section while the NLP subproblem arises for a fixed choice of the boolean variables $W_{n}$.

\subsection{Solution Algorithm}

After solving the preprocessing phase models, the logic-based outer approximation method by Turkay and Grossmann (1996) and its modification by Yeomans and Grossmann (2000b) is applied to solve the GDP problem. The algorithm iterates between the two MILP master problems and the reduced NLP subproblems. The MILP problems are formulated with accumulated linearizations and Big-M constraints, while the NLP subproblem simply arises for fixed configuration $\left(Y_{s}\right)$ and trays $\left(W_{n}\right)$.

In Figure 11, the steps of the solution algorithm are presented. After solving the initialization problem, the section GDP problem is first solved as an MILP problem yielding an optimal sequence configuration by selecting the column sections. The boolean variables that define the existence of sections $\left(Y_{S}=\right.$ true $)$ are used to fix the configuration of the next MILP problem for the optimization of the number of trays which is solved in reduced space by eliminating the false terms in the disjunctions (e.g. equations for nonexistent sections). An NLP model is solved next for the selected configuration and number of trays and feed location. Integer cuts involving the boolean variables related to the existence of sections $Y_{S}$ are then added to solve the next MILP section problem to enforce the selection of a different configuration in the next iteration. The GDP algorithm stops when the there is no improvement in the objective function of the NLP subproblems. It should be noted that global optimality cannot be guaranteed due to the nonconvexities involved in the model. Their effect, however, is reduced with th GDP model since nonexisting trays are not included in the NLP subproblem.

\section{Numerical Examples}

The performance of the algorithm is tested with four examples that are presented in this section. In all cases, final pure products are specified. A constant pressure of 1.01 bar is considered for each column. A feed flow of $10 \mathrm{~mole} / \mathrm{s}$ and saturated liquid products are specified. The thermodynamic properties are taken form Reid et al. (1987). 
Example 1 and 2 involve the separation of n-pentane, n-hexane and n-heptane and uses ideal equilibrium while example 3 and 4 deal with the separation of the azeotropic mixture methanol, ethanol and water and uses ideal gas model for the vapor phase and the Wilson model for the liquid phase. In all cases, the VLE equations involve the transformation of variables suggested by Bauer and Stichlmair (1998) in order to improve the convergence of the NLP subproblems. This transformation yields more linear equations when modeling the VLE equations.

The examples were implemented and solved in GAMS 20.7 (Brooke et al., 1998) in a PIII, $667 \mathrm{MHz}$ with $256 \mathrm{MB}$ of RAM. The code CPLEX 7.5 was used for solving the mixed integer linear programming (MILP) problems and CONOPT2 for the NLP subproblems.

\section{1 Example 1}

A feed of n-pentane (A), n-hexane (B) and n-heptane (C) with molar composition of 0.33/0.33/0.34 is given. The required purity for the products is $98 \%$ for $A, B$ and $C$ with a minimum recovery of $98 \%$ of each product. Each section is assigned a maximum of 15 trays.

The method proposed in this paper was applied to this problem and the solution is reported in Table 1. The preprocessing phase is solved in 2.20 CPU min. The preprocessing solution profiles are shown in Figure 12. The decomposition algorithm is applied next and the optimal solution was found after 5 iterations in 9.25 CPU min. (6.97 min. for the NLP subproblems and $2.29 \mathrm{~min}$. for the MILP subproblems). The total solution time is $11.46 \mathrm{CPU}$ min.

The optimal configuration with a total cost of 140,880 \$/year is shown in Figure 13 (b) (see Table 1). The solution configuration involves the 6 sections selected in the superstructure, as can be seen in Figure 13 (a). Column 1 has 22 trays selected with the feed entering in tray 12, column 2 has 23 trays with a vapor feed entering in tray 14 and column 3 also has also 23 trays and the feed stream in located in tray 10. This scheme can be rearranged in terms of two columns, according to the tray diameters obtained in the solution. Column 1, the stripping section of column 2 and the rectifying section of column 3 have a diameter of $0.45 \mathrm{~m}$, the rectifying section of column 2 has a diameter of $0.6 \mathrm{~m}$ while the stripping section of column 3 has a diameter of $0.63 \mathrm{~m}$ (see Figure 13 (a)). Therefore, the rectifying section of column 2 is moved to the top of column 1 and the stripping section of column 2 is placed on the top of column 3 . In this way, the configuration shown in Figure 13 (b) is obtained. In the optimal rearranged solution, column 1 has 36 trays and the feed stream enters in tray 26. Column 2 has 32 stages, a feed is placed in tray 19 and two liquid product streams are withdrawn from tray 9 and 32. Due to the column coupling, column 2 does not 
have condenser. Column 1 has two condensers, one on the top tray and the other in tray 12. It is interesting to note that the existence of an equipment in an intermediate tray of the column improves the efficiency of the separation scheme since the heat is eliminated in tray 12 at a lower temperature than in the top tray of column 1.

The liquid composition profiles for the optimal configuration of Figure 13 (b) are shown in Figure 14. As can be seen from Figure 14, the separation takes place close to minimum reflux conditions since the composition profiles of column 1 intersect at the feed composition.

The same problem was formulated as a MINLP problem. The formulation of this problem involved the optimization of the feed stream and reboiler location. This representation for tray optimization happens to be the most suitable representation for the superstructure (Barttfeld et al, 2003). The preprocessing phase was also applied to this formulation and DICOPT was used as the solver for this problem giving a relaxed solution with a cost of $140,088 \$$ /year. The problem could not be solved as an integer problem, since no integer feasible solution was found by the solver, even by applying the domain reduction procedure (see Barttfeld et al, 2003) over the binary variables to reduce the size of the problem. The relaxed MINLP solution involves a slightly lower cost than the solution found with the decomposition GDP algorithm. However, since the integer mixed integer solution could not be found, both solutions cannot be compared.

Two conventional alternatives which lead a feasible design for this separation were also optimized, the direct sequence configuration and the side-rectifier configuration. The direct sequence yields a cost of $145,040 \$ / y r$, while the side-rectifier configuration has a total cost of $143,440 \$ / y r$. This fact allows to conclude that the proposed algorithm with $140,880 \$ / y r$ obtained a significantly better solution for this example problem.

\section{2 Example 2}

This example also involves the separation of n-pentane (A), n-hexane (B) and n-heptane (C) but the molar composition considered is $0.6 / 0.2 / 0.2$. The required purity for the products is $98 \%$ for $A, B$ and $C$ with a minimum recovery of $98 \%$ of each product. Each section is assigned a maximum of 15 trays.

The computational results are shown in Table 2. The preprocessing phase is solved in $0.78 \mathrm{CPU}$ min. and the liquid composition profiles for this solution are shown in Figure 15.

The optimal configuration has a total cost of 137,760 \$/year and convergence is achieved in 7 iterations of the algorithm. The total solution time is $13.07 \mathrm{~min}$. $(8.85 \mathrm{~min}$. for the NLP subproblems and $4.22 \mathrm{~min}$. for the MILP subproblems). The optimal solution selects all sections of the superstructure. 
Column 1 has 23 trays activated and uniform diameter of $0.5 \mathrm{~m}$, column 2 has 24 trays and a diameter of $0.62 \mathrm{~m}$ for the rectifying section and $0.39 \mathrm{~m}$ for the stripping section, and column 3 also has 23 trays and a uniform diameter of $0.39 \mathrm{~m}$. This solution can be rearranged in terms of two columns, as shown in Figure 16 (a).

The MINLP formulation was also used to solve this example. In this case as in the previous example, we could not solve the integer problem. However, the relaxed solution for this problem yields a cost of 121,120 \$/year, which is lower than the one achieved applying the GDP decomposition algorithm. Since the MINLP formulation could not be solved as an integer problem no comparisons can be made between the MINLP and GDP solutions.

The direct sequence which is a feasible design for this problem, was optimized yielding a total cost of $138,480 \$$ year which is a higher cost than the one achieved with the proposed method (see Table 2).

\section{3 Example 3}

A feed of methanol (A), ethanol (B) and water (C) with molar composition of 0.5/0.3/0.2 is given. The specifications considered for this problem were taken from Yeomans and Grossmann (2000b), where the required purity for the products is $90 \%$. Each section is assigned a maximum of 20 trays. The superstructure for these example is shown in Figure 17.

The solution found for this problem after applying the decomposition algorithm is reported in Table 3. The preprocessing phase is solved in $6 \mathrm{CPU}$ min. The liquid composition profiles for the columns in the preprocessing phase solution are shown in Figure 18. Note that pure products are obtained in each column since the distillate product of column one crosses the distillation boundary.

The decomposition algorithm is applied next and the optimal solution was found after 3 iterations in 56.1 CPU min. Note that most of the computation time involved is used for solving the NLP subproblems (54.7 min. for the NLP subproblems and $2.33 \mathrm{~min}$. for the MILP subproblems). The total solution time is $62.15 \mathrm{CPU}$ min.

The optimal solution yields a total cost of 321,680 \$/year (see Table 3). Both sections of column 1 and 2 were selected from the initial superstructure. Column 1 has 40 trays with a diameter of $0.70 \mathrm{~m}$. Column 2 has 39 trays with diameters of 0.70 and $0.55 \mathrm{~m}$ for the rectifying and stripping sections, respectively. This solution can be rearranged in terms of one column and a side stripper as shown in Figure 19. The rectifying section of the original column 2 was placed on the top of the first column since both have the same diameter. The main feed enters in tray 40 of column 1 and product is withdrawn form 
this column in tray 20 to provide part of the main product $P P_{1}$ (see Figure 19). Note that the product rich in methanol (A) is formed by the contributions of both the liquid top product of columns 1 and 2 . Note that the liquid top product emerging from column 1 top has a higher purity $(98.9 \%$ of $A$ ) than the one required in the final product, but this stream is mixed with the liquid product withdrawn in tray 20 of column $1(51 \%$ of $A)$ to meet the required purity of $90 \%$. This situation is shown in Figure 20, where the liquid composition profiles of the configuration of Figure 19 are presented.

It is worth noting that the optimal configuration found by the algorithm does not produce products with the azeotropic composition. This is because of the required purity of the products. As can be seen in Figure 20, column 1 separates the feed into a distillate rich in water (90\%) and a top product which is located on a different distillation region than the original feed. This product enters column 2 to be separated into a top product with high purity in methanol and into a bottom product which already achieved the required purity of methanol. It should be noted that the separation is performed close to minimum reflux conditions since the columns involve a large number of trays.

\section{4 Example 4}

Another example involving a higher purity requirement for the products was solved for the same feed composition in example 3. In this case, a purity of 95\% was specified for the system in Example 2.

The optimal configuration is shown in Figure 21 where column 1, 2 and 3 were selected form the initial superstructure. This solution was found in 3 iterations of the decomposition algorithm and the recycle of a stream with the azeotrope composition in order satisfy the products purity. The total solution time is 46 CPU minutes (36.26 CPU min. for the NLP subproblems, 3.70 CPU min. for the MILP subproblems and 6 CPU min. to solve the preprocessing phase). Column 1 involves 39 trays and a diameter of $0.65 \mathrm{~m}$, column 2 has 38 trays and a diameter of $0.56 \mathrm{~m}$ while column 3 has 35 trays and a diameter of $0.32 \mathrm{~m}$. In Figure 22, the liquid composition profiles for the optimal configuration are presented.

Finally, the use of intermediate condensers in column 1 were analyzed. In Figure 23, the preprocessing solution for a feed composition of $0.3,0.4$ and 0.3 of methanol, ethanol and water, respectively, is shown. In part (a) of Figure 23, column 1 has only one condenser located in the top tray while the profiles shown in Figure 23 (b) were obtained by placing four intercondensers in column 1 on trays 1, 3, 5 and 10. Column 1 of Figure 23 (b) requires less energy than column 1 in Figure 23 (a). Both configurations achieved pure products. However, the structure without intercondensers has a column 1 
profile which crosses more deeply into the distillation boundary than the scheme with intercondensers. The composition profile of Figure 23 (a) finishes at the composition 0.594, 0.381, 0.024 of methanol, ethanol and water, respectively while the profile of Figure 23 (b) finishes at the composition 0.574, 0.40 and 0.026. This fact produces $3 \%$ of savings in the total energy of the process. According to our experience, we can conclude that the use of intercondensers allows crossing the distillation boundary and locates the distillate composition closer to the minimum composition that has to be reached for crossing the boundary and achieving pure products. It is interesting to note that small perturbations in the composition space after crossing the distillation boundary require large amounts of energy.

\section{Conclusions}

This paper has presented an optimization procedure for the synthesis of complex distillation configurations. A superstructure using tray-by-tray columns and based on the reversible model was considered. The problem was formulated as a GDP model, where boolean variables related to the existence of column sections and individual trays existence were defined.

A decomposition algorithm was proposed to solve the problem. The problem is decomposed into two levels of decisions and is solved in an iterative procedure. The algorithm includes an initialization phase where the reversible sequence is approximated. This initialization phase enhances the robustness and convergence of the economic formulations.

Numerical examples were solved to test the performance of the formulations. Two zeotropic examples were solved and nontrivial configurations were found, which include column coupling. In the azeotropic example, the influence of the product purity specification was analyzed with respect to the azeotrope recycle. Also, the influence of including intercondensers in the first column was analyzed.

In all the examples, the solutions were obtained with the proposed method are non trivial and require reasonable solution times.

\section{References}

Agrawal R. (1996). Synthesis of Distillation Column Configurations for Multicomponent Separations. 1996. Ind. Eng. Chem. Res. 35, 1059.

Annakou O; Mizsey P. (1996). Rigorous Comparative Study of Energy-Integrated Distillation Schemes. Ind. Eng. Chem. Res. 35, 1877. 
Barttfeld M.; Aguirre P. A. (2002). Optimal Synthesis of Distillation Processes. Part I. Preprocessing Phase for Zeotropic Mixtures. Ind. Eng. Chem. Res. 41, 5298.

Barttfeld M.; Aguirre P. A.; Grossmann I. E. (2003) Alternative Representations and Formulations for the Economic Optimization of Multicomponent Distillation. Comp. Chem. Eng. 27, 363-383.

Barttfeld M.; Aguirre P. A. (2003). Optimal Synthesis of Multicomponent Zeotropic Distillation Processes. Part II. Preprocessing Phase and Rigorous Optimization of Efficient Sequences. To appear in Ind. Eng. Chem. Res.

Brooke A.; Kendrick D.; Meeraus A.; Raman R. GAMS, A User Guide. 1998.

Bauer M. B.; Stichlmair J. (1998). Design and Economic Optimization of Azeotropic Distillation Processes Using Mixed-Integer Nonlinear Programming. Comp. Chem. Eng. 22, 9, 1271.

Caballero J. A.; Grossmann I. E. Thermodynamically Equivalent Configurations for Thermally Coupled Distillation Columns. Submitted for publication (2002).

Dunnebier G.; Pantelides C. C. (1999). Optimal Design of Thermally Coupled Distillation Columns. Ind. Eng. Chem. Res. 38, 162.

Fidkowski, Z; Krolikowski L. (1986). Thermally Coupled System of Distillation Columns: Optimization Procedure. AICHE J. 32, 537.

Fidkowski Z.; Agrawal R. (1995). Utilization of Waste Heat Steam in Distillation. Ind. Eng. Chem. Res. 34, 1287.

Fidkowski Z.; Agrawal R. (1996). On the Use of Intermediate Condensers in the Rectifying Section and Reboilers in the Stripping Section. Ind. Eng. Chem. Res. 35, 2801.

Fonyó Z. (1974). Thermodynamic Analysis of Rectification I. Reversible Model of Rectification. Int. Chem. Eng. 14 (1), 18.

Glinos K.; Malone F. (1988). Optimality Regions for Complex Column Alternatives in Distillation Systems. Chem. Eng. Res. Des. 66, 229.

Koehler J.; Aguirre P. A.; Blass E. (1992). Evolutionary Thermodynamic Synthesis of Zeotropic Distillation sequences. Gas Sep. Purif. 6 (3), 153.

Kondili E.; Pantelides C. C.; Sargent R. W. H. (1993). A General Algorihm for Short-Term Scheduling of Batch Operations - I: MILP Formulation. Comp. Chem. Eng. 17, 211.

Reid R. C.; Prausnitz M. P.; Poling B. E. The Properties of Gases and Liquids; McGraw-Hill: New York, 1987.

Sargent R. W. H.; Gaminibandara K. Optimal Design of Plate Distillation Columns. In Optimization in Action; Dixon, L. C. W., Ed; Academic Press: New York, 1976. 
Sargent R. W. H. A (1998). Functional Approach to Process Synthesis and its Application to Distillation Systems. Comp. Chem. Eng. 22, 31.

Stichlmair J. G.; Fair J. R. Distillation. Principles and Practice. 1998, New York:Wiley-VCH.

Triantafyllou C.; Smith R. (1992). The Design and Optimization of Fully Thermally Coupled Distillation Columns. Trans. Inst. Chem. Eng. 70, 118.

Turkay M.; Grossmann I. E. (1996). Logic-based MINLP Algorithm for the Optimal Synthesis of Process Networks. Comp. Chem. Eng. 20, 959.

Yeomans H.; Grossmann I. E. (1999). Nonlinear Disjunctive Programming Models for the Synthesis of Heat Integrated Distillation Sequences. Comp. Chem. Eng. 23, 1135.

Yeomans H.; Grossmann I. E. (2000a). Disjunctive Programming Models for the Optimal Design of Distillation Columns and Separation Sequences. Ind. Eng. Chem. Res. 39, 1637.

Yeomans H.; Grossmann I. E. (2000b). Optimal Design of Complex Distillation Columns Using Rigorous Tray-by-Tray Disjunctive Programming Models. Ind. Eng. Chem. Res. 39, 4326.

\section{Nomenclature}

$\begin{array}{cl}\text { Ccool } & \text { cooling water cost } \\ \text { Csteam } & \text { steam cost } \\ \text { Cinv } & \text { investment cost } \\ \text { Cop } & \text { operating cost } \\ \text { Ctray } & \text { tray cost in section } s \\ \text { Ccol }_{S} & \text { column shell cost in section } s \\ \text { Ccond }_{k} & \text { condenser cost in column } k \\ \text { Creb }_{k} & \text { reboiler cost in column } k \\ \text { Dcol }_{n} & \text { Diameter of tray } n \\ \text { Fo }_{n} & \text { feed flow } \\ f_{n, i}^{L} & \text { fugacity in the liquid phase of component } i \text { in tray } n \\ f_{n, i}^{L} & \text { fugacity in the vapor phase of component } i \text { in tray } n \\ h f e e d_{n f, n} & \text { enthalpy of the feed } n f \text { entering tray } n \\ h l i q_{n} & \text { enthalpy of the liquid stream leaving tray } n \\ h v a p_{n} & \text { enthalpy of the vapor stream leaving tray } n \\ h p l i q_{n} & \text { enthalpy of liquid product leaving tray } n \\ h p v a p_{n} & \text { enthalpy of vapor product leaving tray } n \\ h P_{j} & \text { enthalpy of product } j \\ & \end{array}$




$\begin{array}{cl}i & \text { component } \\ k & \text { set denoting each column of the superstructure } \\ L_{n} & \text { total liquid flow emerging from tray } n \\ m v_{k} & \text { mole flow of steam in column } k \\ m w_{k} & \text { mole flow of steam in column } k \\ n t r a y_{S} & \text { number of trays of section } s \\ P_{n} & \text { Pressure of tray } n \\ P h_{j} & \text { total enthalpy of product } j \\ P L_{n} & \text { liquid product flow emerging column } n \\ P V_{n} & \text { vapor product flow emerging column } n \\ P P_{j} & \text { flow of final product } j \\ P z_{j, i} & \text { individual product flow of product } j \text { in component } i \\ Q_{n} & \text { energy exchanged in tray } n \\ s t g_{n} & \text { counter for the existence of a tray } n \\ T_{n}^{L} & \text { Temperature of the liquid phase in tray } n \\ T_{n}^{L} & \text { Temperature of the vapor phase in tray } n \\ V_{n} & \text { total vapo flow emerging from tray } n \\ W_{n} & \text { boolean variable denoting the existence of a tray } n \\ x_{n, i} & \text { mole fraction of component } i \text { in tray } n \text { in the liquid phase } \\ y_{n, i} & \text { mole fraction of component } i \text { in tray } n \text { in the vapor phase } \\ Y_{s} & \text { boolean variable denoting the existence of a section } s \\ z f_{n, i} & \text { mole fraction of component } i \text { in feed stream entering tray } n \\ z f_{i} & \text { mole fraction of component } i \text { in feed stream } \\ \varepsilon_{P j, i} & \text { mole fraction of component } i \text { in product } j \\ & \text { recovery fraction of component } i \\ & \text { purity of component } i \\ & \end{array}$

\section{Acknowledgments}

The authors want to thank for the financial support from CONICET (Consejo Nacional de Investigaciones Científicas y Técnicas), ANPCYT (Agencia Nacional de Promoción Científica y Técnica), UNL (Universidad Nacional del Litoral) from Argentina and the Center for Advanced Process Decision-making at Carnegie Mellon. 


\section{Appendix A: Initialization Phase of the Decomposition Algorithm}

In this section, the sequence preprocessing phase is briefly described. The procedure for zeotropic mixtures is first described and extensions for azeotropic cases are then outlined.

\section{Zeotropic Case}

In the preprocessing phase the reversible distillation sequence is adiabatically approximated. In this phase an initial solution for the economic problem is generated.

This preliminary phase involves the solutions of two types of problems. In a first step, overall mass and energy balances are formulated as NLP problems to compute the reversible products in each single unit. In this step, the primary and secondary products are specified to have the exhausting pinch compositions flows and compositions in order to efficiently integrate the columns (Barttfeld and Aguirre, 2003). For that reason, in a second step the reversible exhausting pinch point composition is calculated using the information of the reversible products computed previously. Then, a problem is solved to generate initial values related to the sections integration in the last level of the superstructure. This formulation computes the connection flows and energy demands after the integration of the column take place. These preliminary formulations are well-behaved problems that compute initial values and bounds for the rigorous preprocessing NLP tray-bytray formulation. The formulations as well as an algorithmic procedure to sequentially solve these problems are described in detail in Bartfeld and Aguirre (2003).

After these problems are solved, initial values to thermodynamically optimize the superstructure of Figure 9 are available. Then, the RDSM-based sequence is optimized to approximate reversible conditions. Some of the characteristics of this problem are outlined next:

- All the columns of the superstructure are fixed. It means that all sections and all trays are selected and no structural optimization (discrete decisions) takes place.

- In order to approximate reversibility conditions, the columns of the superstructure have the number of trays fixed at the upper bound.

- The separation task performed in each column is the reversible or preferred separation (Koehler et al., 1992; Fonyó, 1974; Barttfeld and Aguirre, 2002; Stichlmair and Fair, 1998).

- The main products are specified to have the exhausting pinch point composition (Barttfeld and Aguirre, 2003). The secondary feed are specified to be in equilibrium with the main products. 
- Heat exchange is allowed in all columns despite the fact that integration between columns take place. As a consequence, the energy can be exchanged at intermidiate levels of the superstructure, leading to more efficient designs.

Because good initial values and bounds are available for this problem its convergence is enhanced. Thus, a good initial guess, which is a feasible solution for the economic problem is generated.

This rigorous tray-by-tray problem approximates the reversible separation task in each column is described next. The objective function is given by equation (46):

$$
\begin{aligned}
z= & \sum_{i=1}^{N C} \sum_{k=1}^{K}\left(y_{\text {top }_{k}, i}-y_{\text {top }_{k}, i}^{\text {pinch }}\right)^{2}+\sum_{k=1}^{K}\left(x_{\text {bot }_{k}, i}-x_{\text {bot }_{k}, i}^{\text {pinch }}\right)^{2}+\sum_{\substack{k=1 \\
k \text { even }}}^{K}\left(z f_{S_{k}, i}-x_{\text {top }_{k}, i}^{\text {pinch }}\right)^{2}+ \\
& \sum_{\substack{k=1 \\
\text { kodd }}}^{K}\left(z f_{S_{k}, i}-y_{\text {bot }_{k}, i}^{\text {pinch }}\right)^{2}+\sum_{j=1}^{J}\left(z P_{j, i}-x p_{j, i}\right)^{2}
\end{aligned}
$$

Equation (46) expresses the difference between each stream composition which connect the units respect to the reversible exhausting pinch point composition. The first (second) term of equation (46) specifies the vapor (liquid) composition $y_{t o p_{k}, i}\left(x_{t o p_{k}, i}\right)$ exiting from the top (bottom) of a column to have the composition of the vapor (liquid) emerging from the reversible rectifying (stripping) exhausting pinch point zone $y_{t o p_{k}, i}^{\text {pinch }}\left(x_{t o p_{k}, i}^{\text {pinch }}\right)$ (see Figure 2). By including the third (fourth) term in equation (46), the liquid (vapor) secondary feed composition $z f_{S_{k}, i}$ will be similar to the liquid (vapor) composition $x_{\text {top }_{k}, i}^{\text {pinch }}\left(y_{\text {bot }_{k}, i}^{\text {pinch }}\right)$ entering in the reversible rectifying (stripping) exhausting pinch point zone. Then, the liquid and vapor streams connecting columns where multicomponent separations take place have the composition and flow of the reversible exhausting pinch point. Note that the pinch point compositions were previously computed by solving the auxiliary NLP problems mentioned before. Then, these compositions are parameters in the tray-by-tray NLP model which approximates the reversible sequence in the preprocessing phase. The final products are also specified in the fifth term of the objective function. In this term, $x p_{j, i}$ is the desired composition of the final product $j$.

The constraints of the rigorous pre-processing problem are given by equations (10) to (31) and the following two extra constraints:

$$
\begin{aligned}
& P L_{t o p_{k}}=F_{F_{2 k+1, n}} \quad 1 \leq k \leq K-N C-1, k \in\left\{\sum_{r=1}^{m} 2^{r-1}\right\}, 1 \leq m \leq N C-1 \\
& P L_{t o p_{k}}=0 \quad 2 \leq k \leq k-N C-1
\end{aligned}
$$


Equations (47) impose the condition that the flow from the bottom of a column becomes the feed of a next column and it does not contribute to form the final product $P P_{J}$. In Figure 9, note that this condition requires that the bottom product flow that emerges from column 1 is fed to column 3 . No flow contributing to the product $P P_{J}$ is allowed. This condition is imposed in the preprocessing problem because every column has a number of trays fixed in the upper bound. It means that no structural optimization is considered in this phase and the problem is solved keeping all the columns of the initial superstructure. Equation (48) models the analogous situation for the top products, requiring that no liquid product emerge from any top tray. Since no sections are eliminated in this phase, the final product $P P_{1}$ is formed just with the contribution of the top product of column $2^{N C-2}$ (see Figure 9).

Azeotropic Case

The preprocessing procedure presented above can be easily extended for ternary azeotropic mixtures containing a composition diagram similar to the one in Figure 8. The main difference in the scheme in this case relies on the fact that due to the existence of a distillation boundary, the distillate product of the first column is given by a ternary mixture. Then, the reversible product composition and flows cannot be computed by overall mass and energy balances as in the zeotropic case. Moreover, since the distillate product of column 1 has the same number of components as the feed stream, the reversible exhausting point does not take place in the rectifying section of column 1. For that reason, for azeotropic mixtures, a preprocessing phase for column 1 as a single unit has to be included. In this phase, the single column is modeled as a trayby-tray model to approximate reversible conditions. Details on this procedure can be found in our previous work (Barttfeld et al., 2003).

After solving the single column preprocessing phase for column 1, the composition of the ternary distillate product $y_{t o p_{1}, i}^{\text {rever }}$ is known. As was shown in our previous work (Barttfeld et al., 2003), this composition crosses the distillation boundary. Then, the objective function for the azeotropic case is given by the following expression:

$$
\begin{aligned}
z= & \sum_{i=1}^{N C}\left(y_{\text {top }_{1}, i}-y_{\text {top }_{1}, i}^{\text {rever }}\right)^{2}+\sum_{k=2}^{K}\left(y_{\text {top }_{k}, i}-y_{\text {top }_{k}, i}^{\text {pinch }}\right)^{2}+\sum_{k=1}^{K}\left(x_{\text {bot }_{k}, i}-x_{\text {bot }_{k}, i}^{\text {pinch }}\right)^{2}+ \\
& \sum_{\substack{k=2 \\
k \text { even }}}^{K}\left(z f_{S_{k}, i}-x_{\text {top }_{k}, i}^{\text {pinch }}\right)^{2}+\sum_{\substack{k=1 \\
\text { kodd }}}^{K}\left(z f_{S_{k}, i}-y_{\text {bot }_{k}, i}^{\text {pinch }}\right)^{2}+\sum_{j=1}^{J}\left(z P_{j, i}-x p_{j, i}\right)^{2}
\end{aligned}
$$

Note that equation (49) only differs from equation (46) in the term formulated for the top product exiting column 1. The difference between the distillate product composition $y_{t_{0 p}, i}$ from column 1 and the reversible composition $y_{t o p_{1}, i}^{\text {rever }}$ is minimized. Also note that there is no secondary feed in the rectifying section of column 
1 since the exhausting pinch point does not take place. The constraints of the problem are the same than in the zeotropic case. 


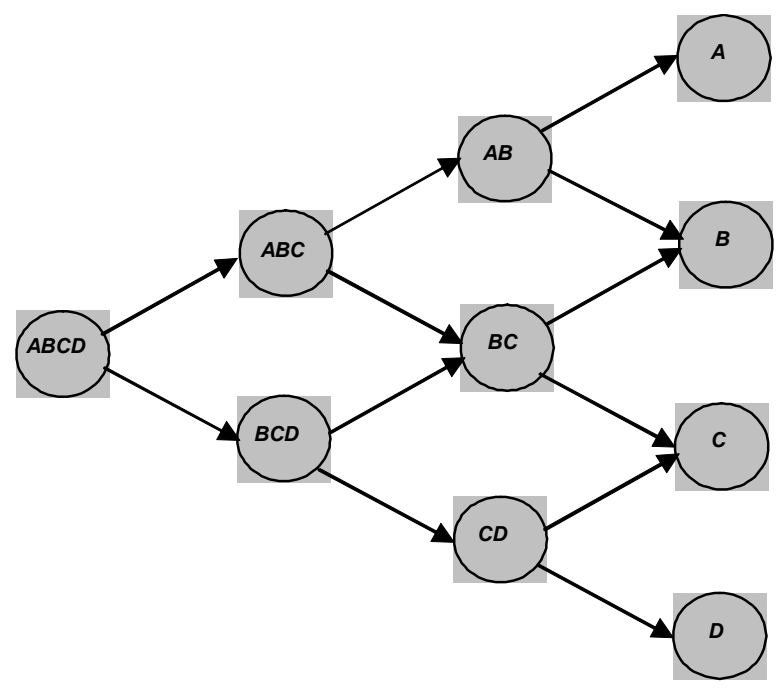

Figure 1: Sargent-Gaminibandara STN representation for a four component zeotropic mixture

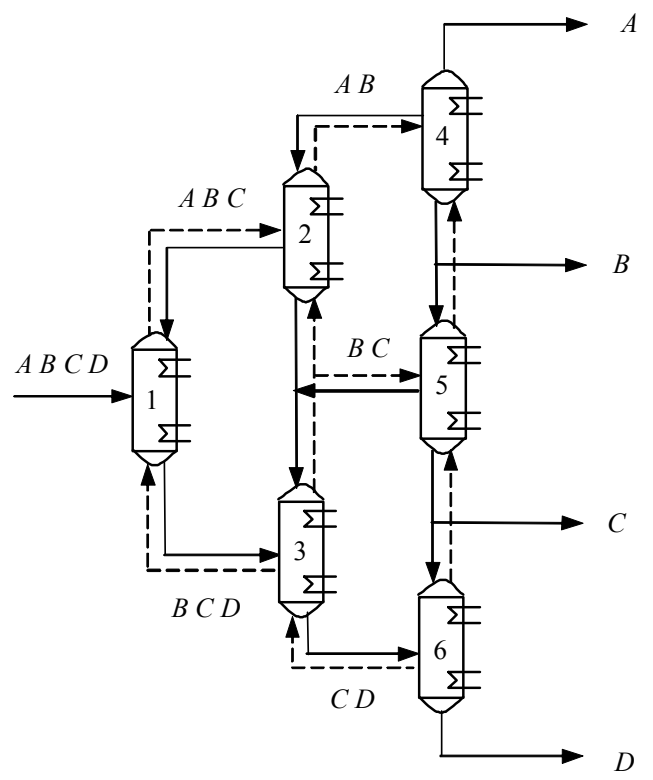

(a)

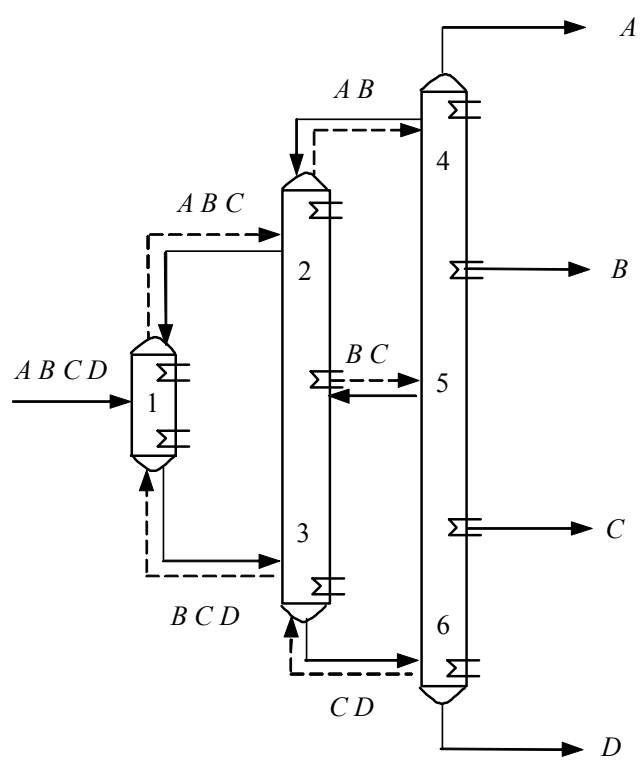

(b)

Figure 2: Sargent-Gaminibandara superstructure. 


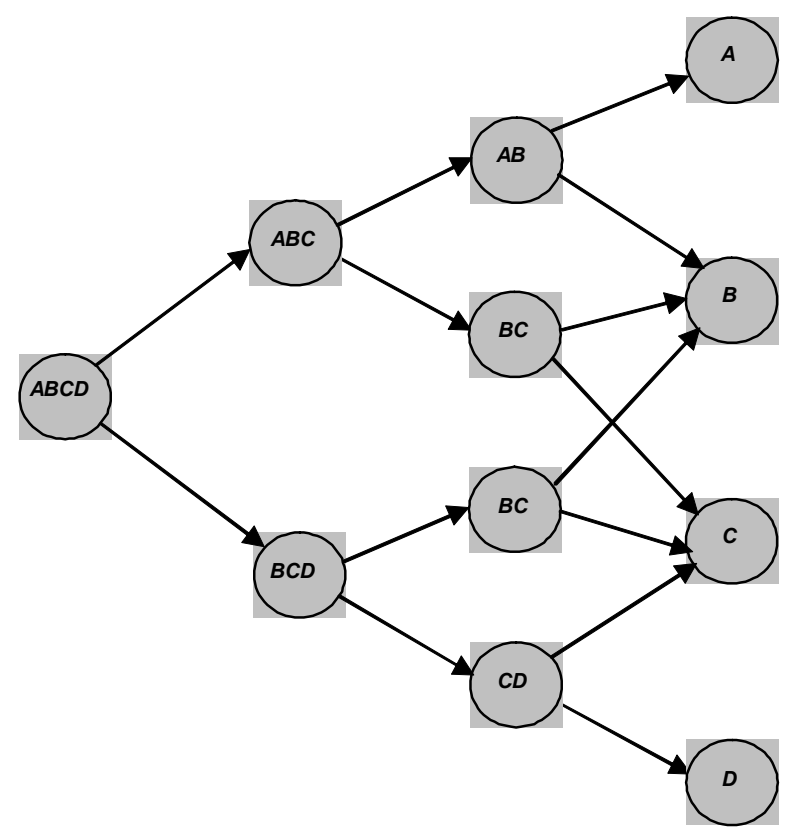

Figure 3: RDSM STN representation

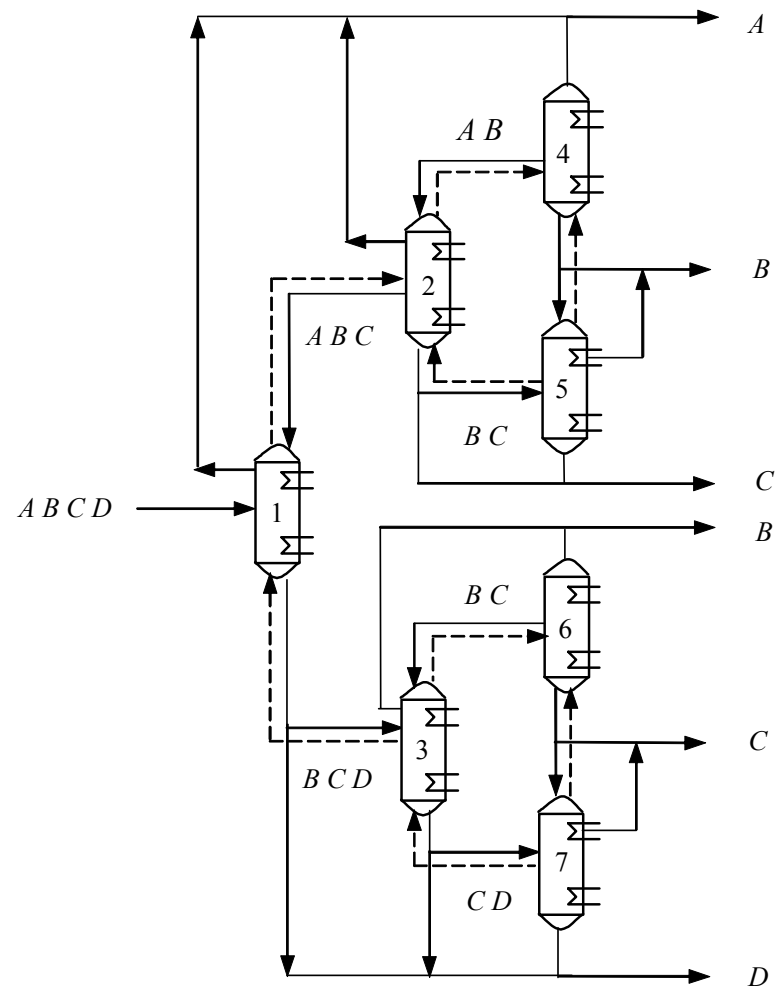

(a)

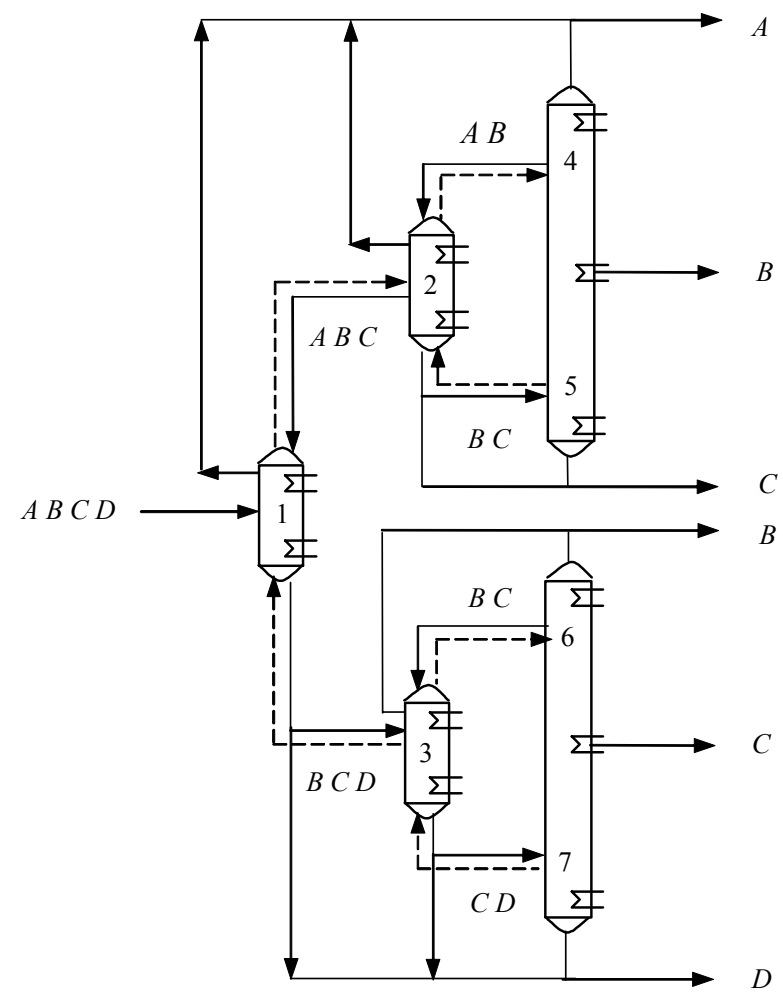

(b)

Figure 4: RDSM superstructure for a four component mixture 


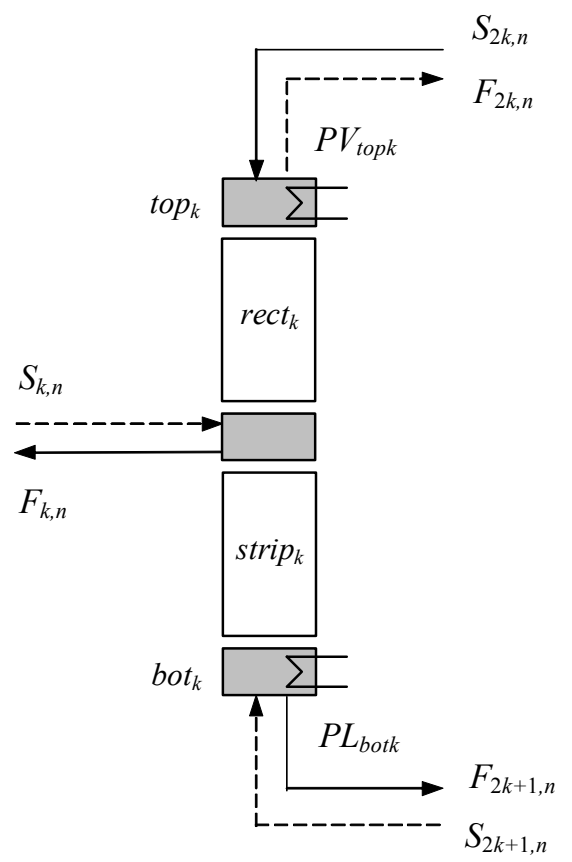

Figure 5: Single column superstructure.

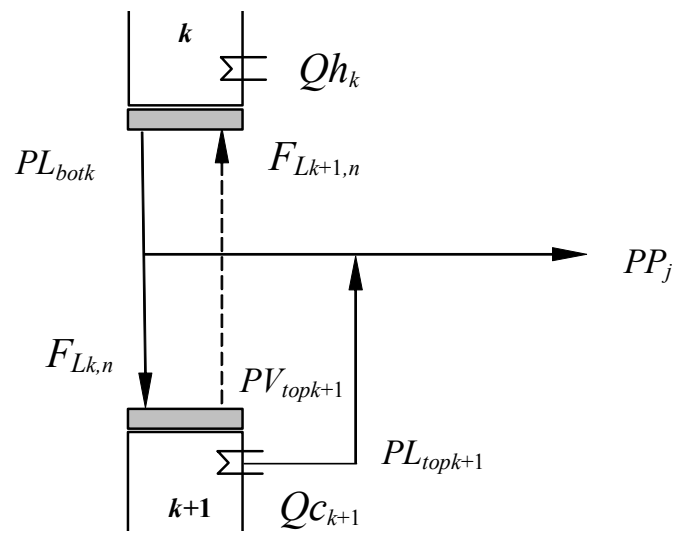

Figure 6: Column integration superstructure. 


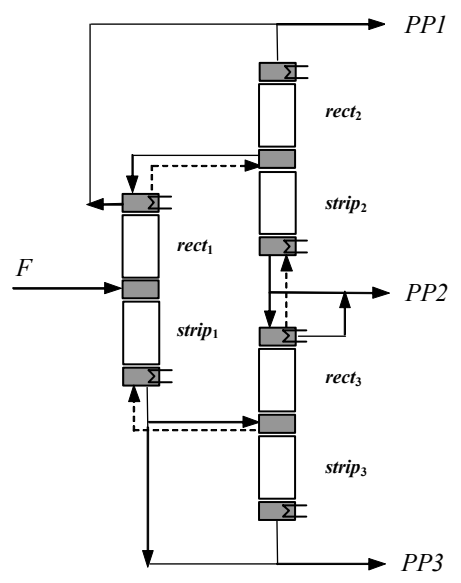

(a)

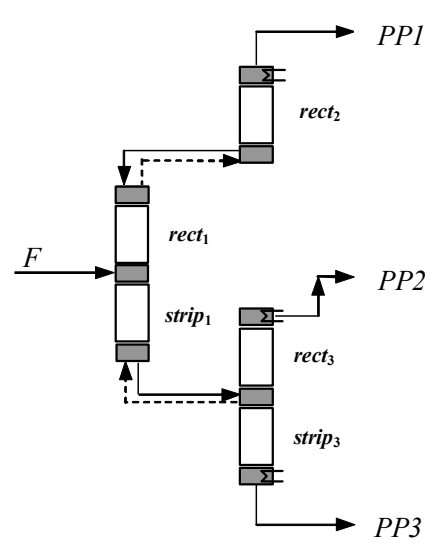

(b)

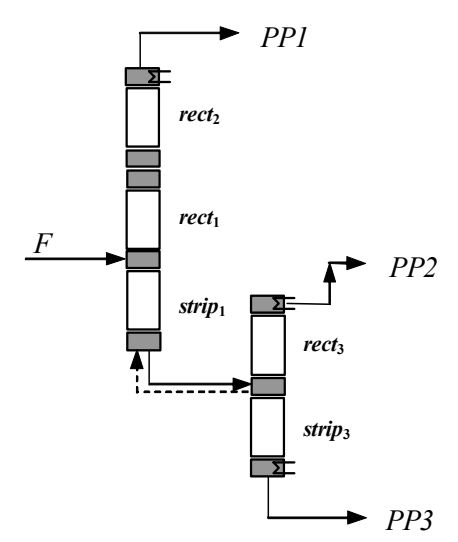

(c)

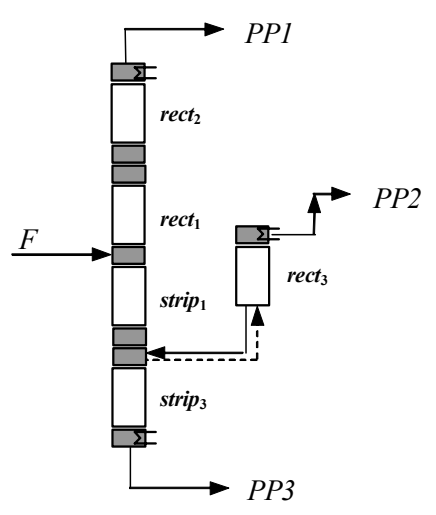

(d)

Figure 7: Superstructure and configurations for a ternary zeotropic system 


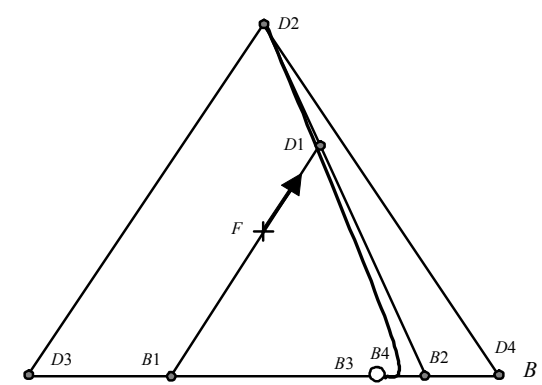

(a)

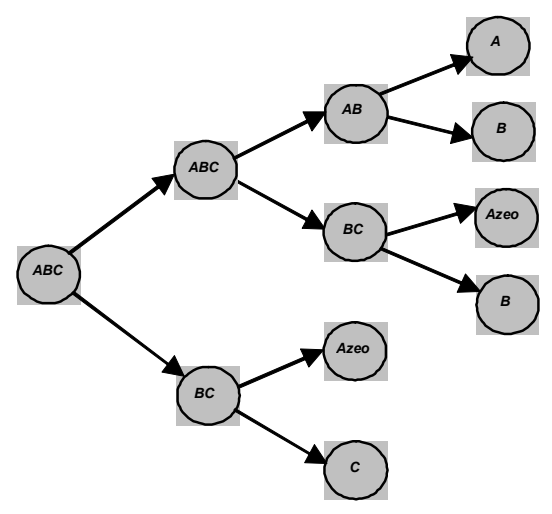

(b)

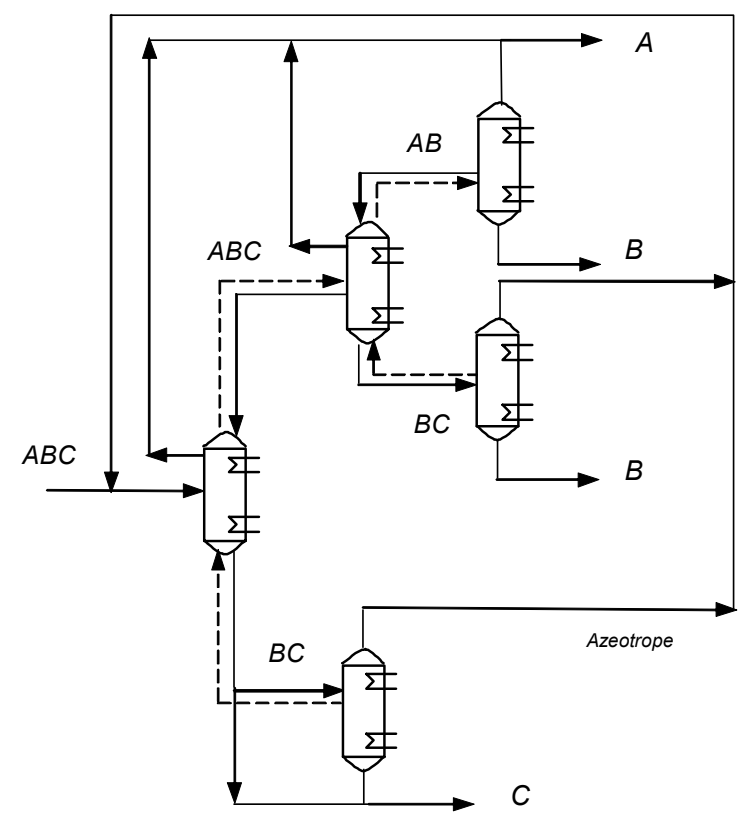

(c)

Figure 8: Azeotropic STN Representation and Superstructure 


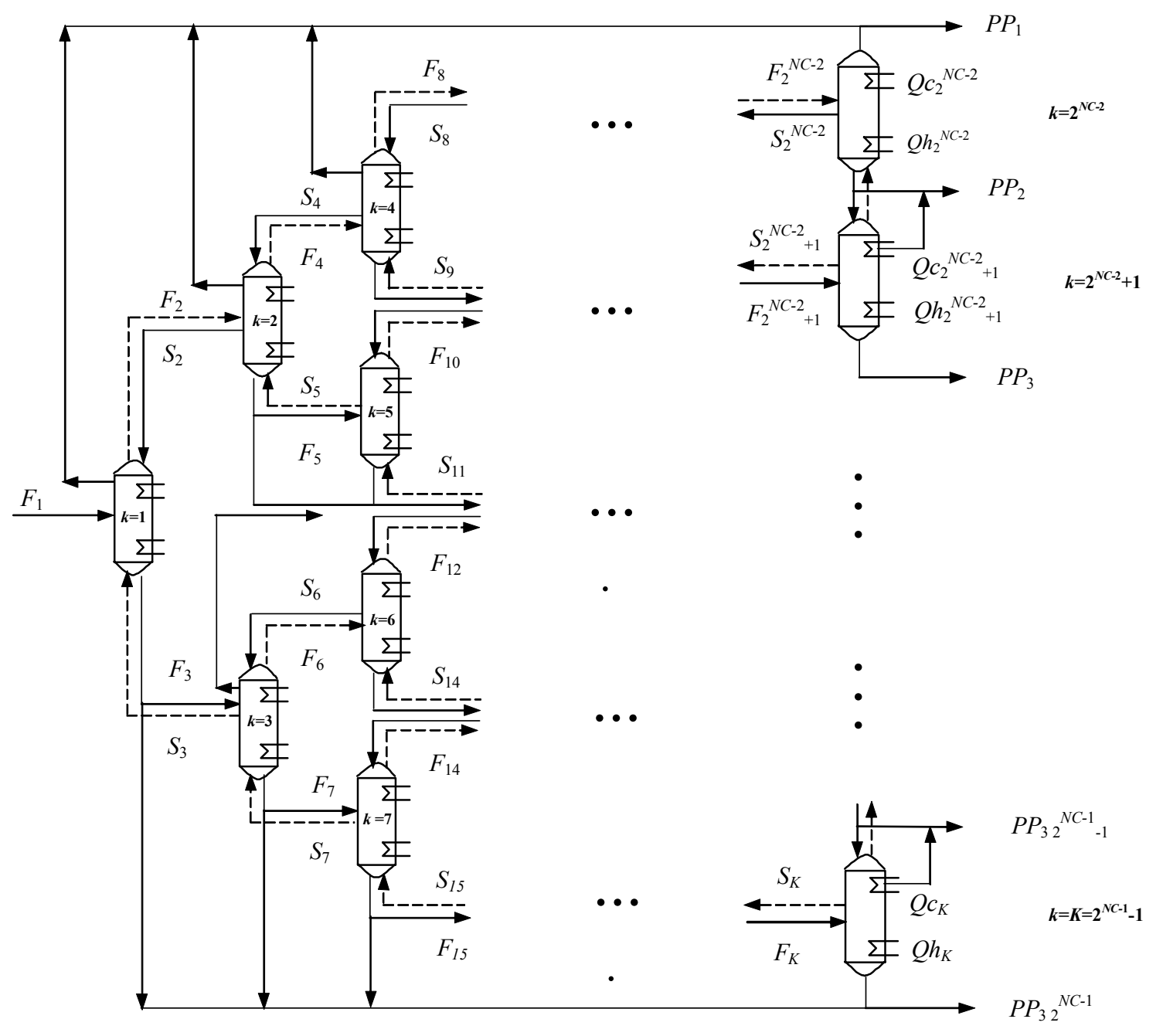

Figure 9: RDSM superstructure for an $N C$-component mixture 


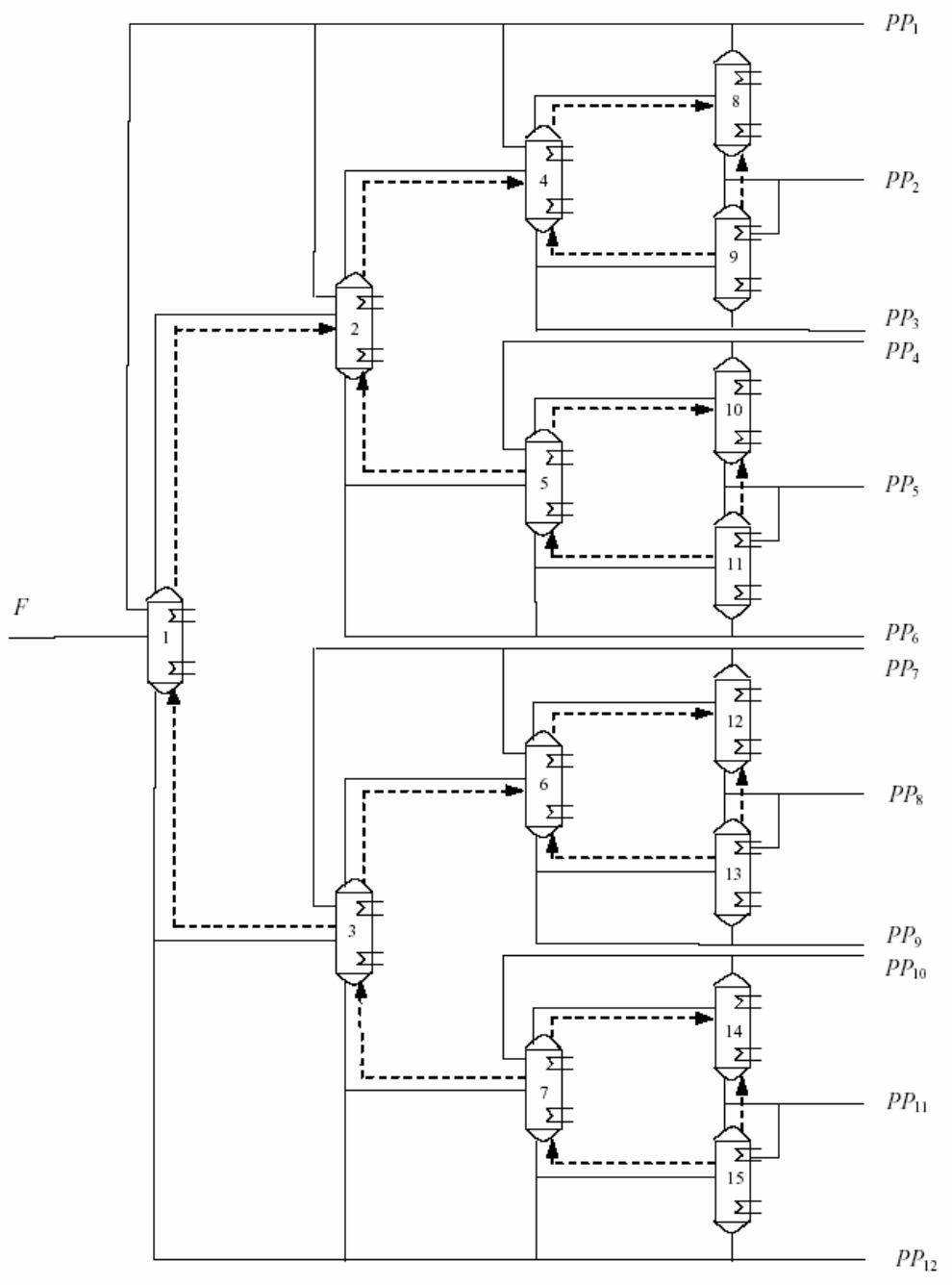

Figure 10: Superstructure for a five components feed 


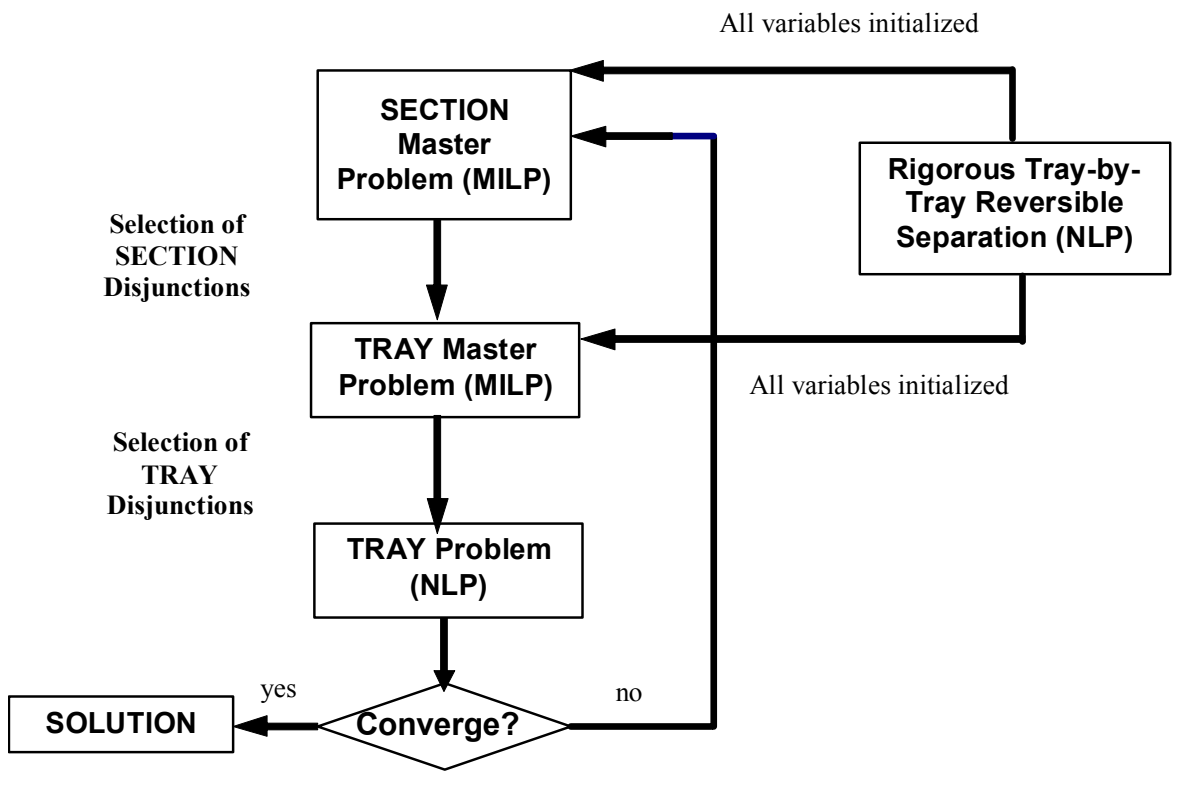

Figure 11: Decomposition algorithm

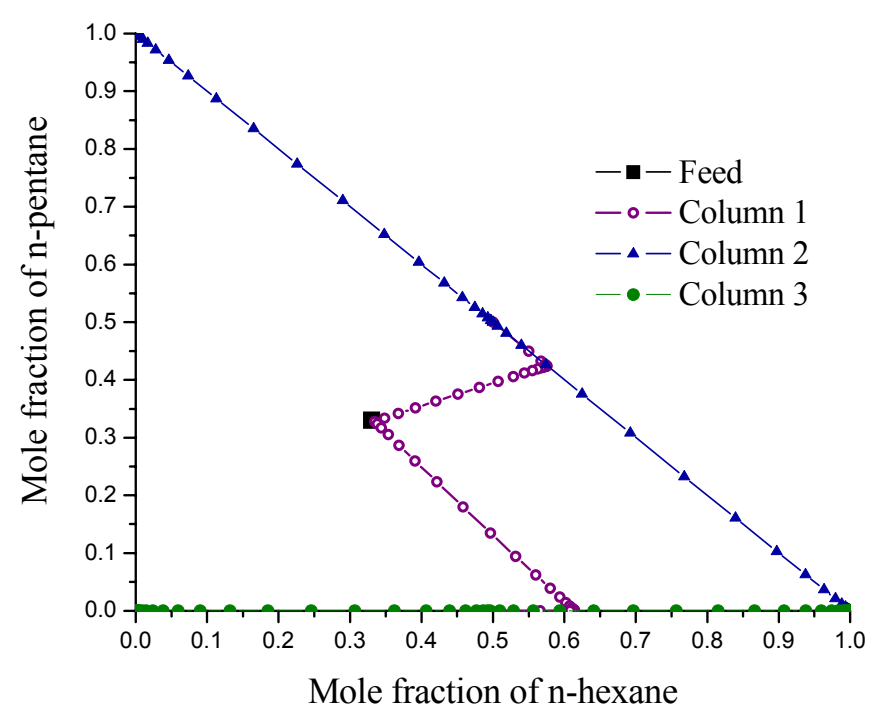

Figure 12: Example 1 - Preprocessing solution liquid profiles 


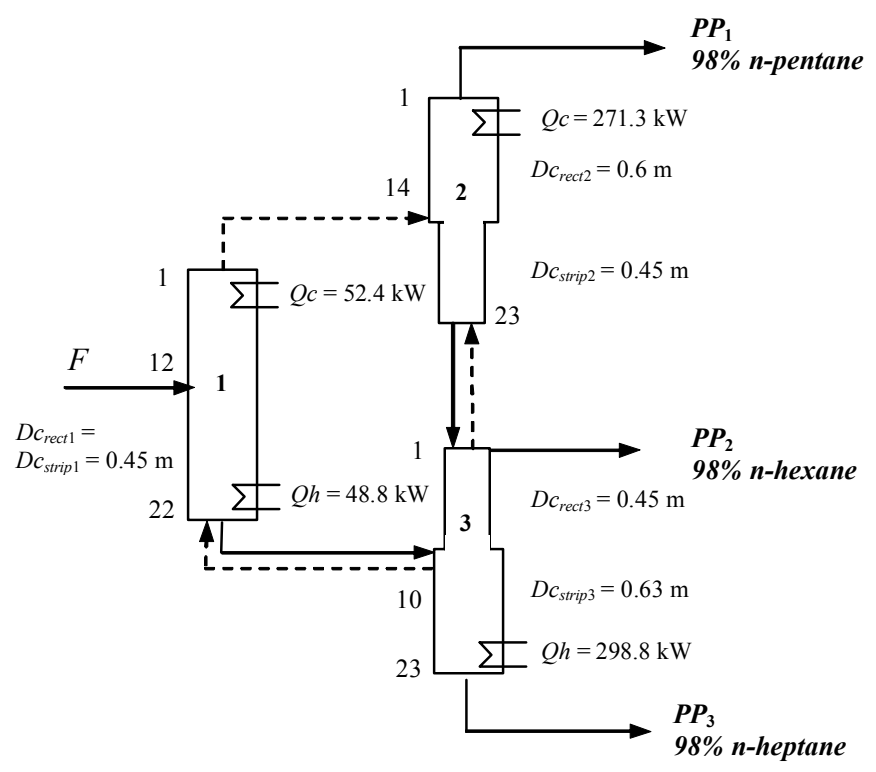

(a)

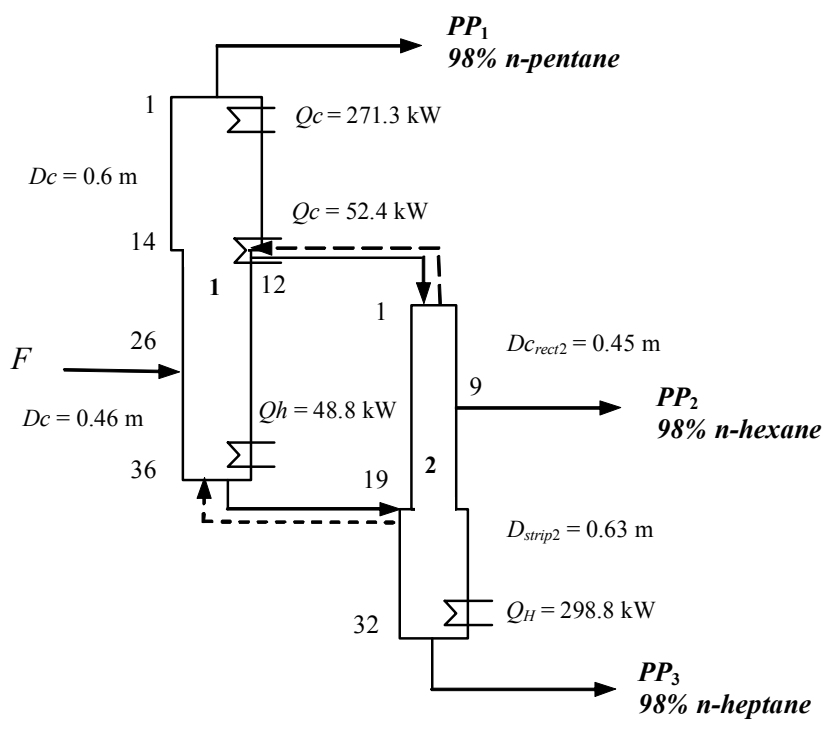

(b)

Figure 13: Example 1 - (a) Superstructure with selected sections. (b) Optimal Configuration in terms of two columns.

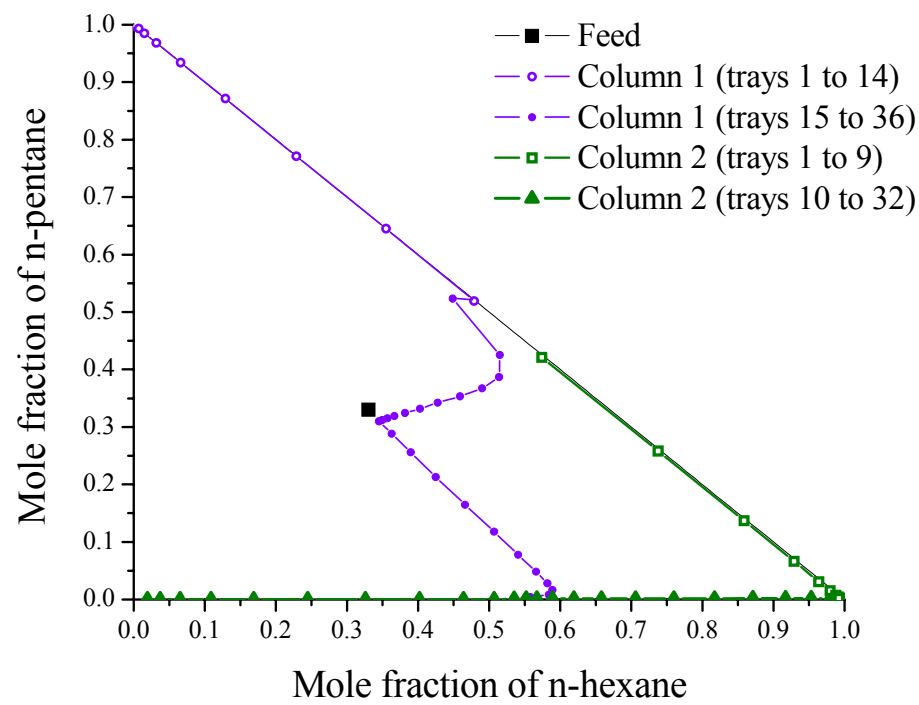

Figure 14: Liquid composition profiles of the optimal configuration 


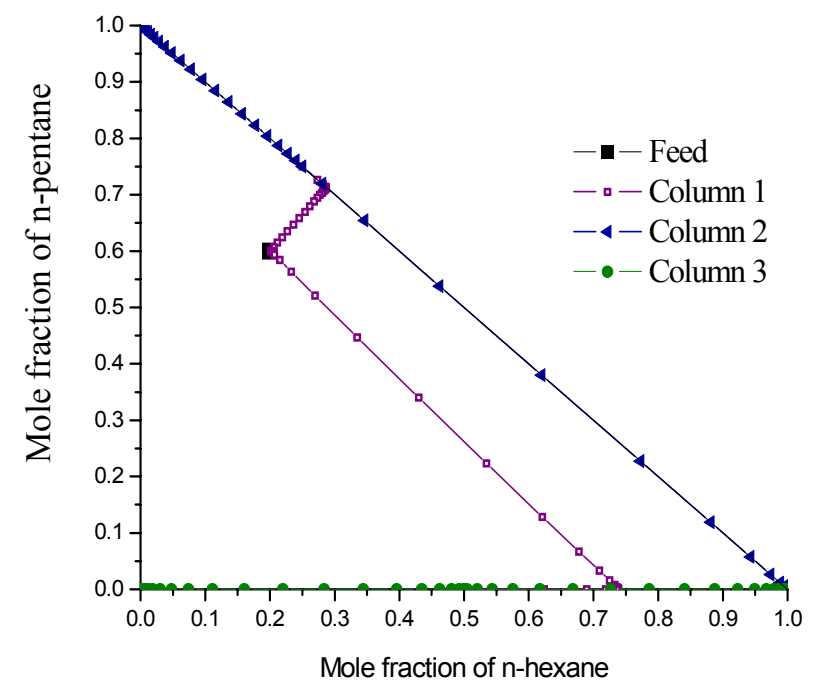

Figure 15: Example 2 - Preprocessing solution liquid profiles

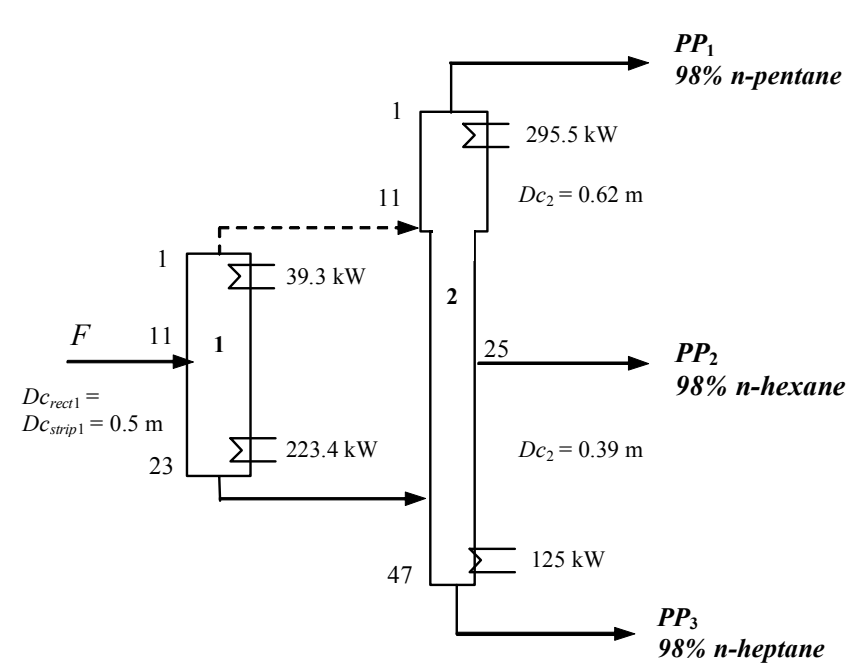

(a)

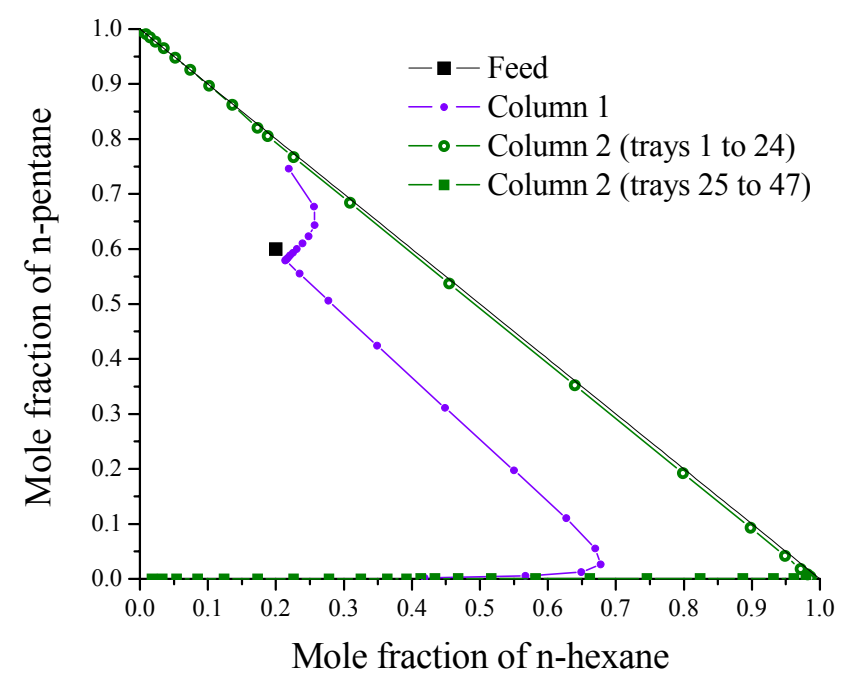

(b)

Figure 16: Example 2 - (a) Optimal Configuration. (b) Liquid composition profiles of the optimal configuration 


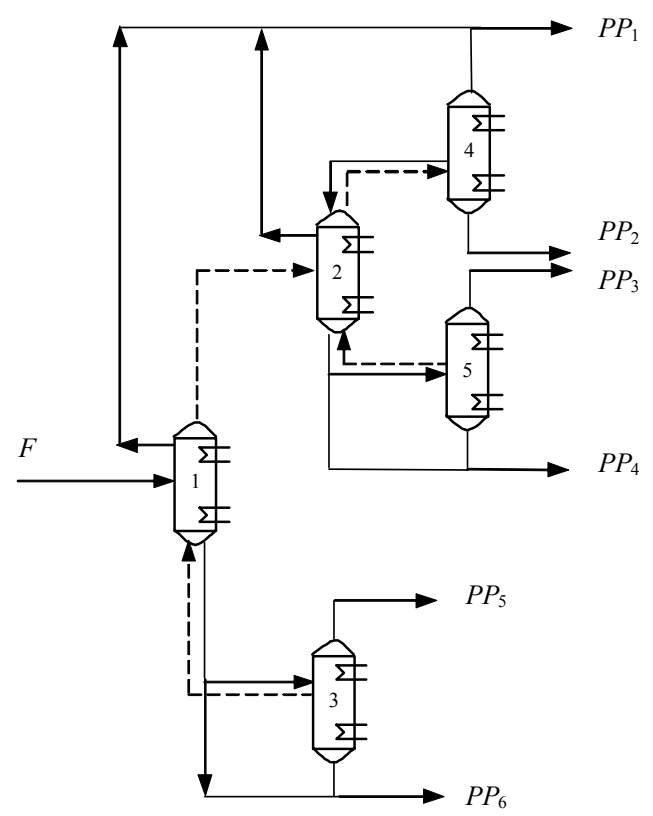

Figure 17: Example 3 - Superstructure

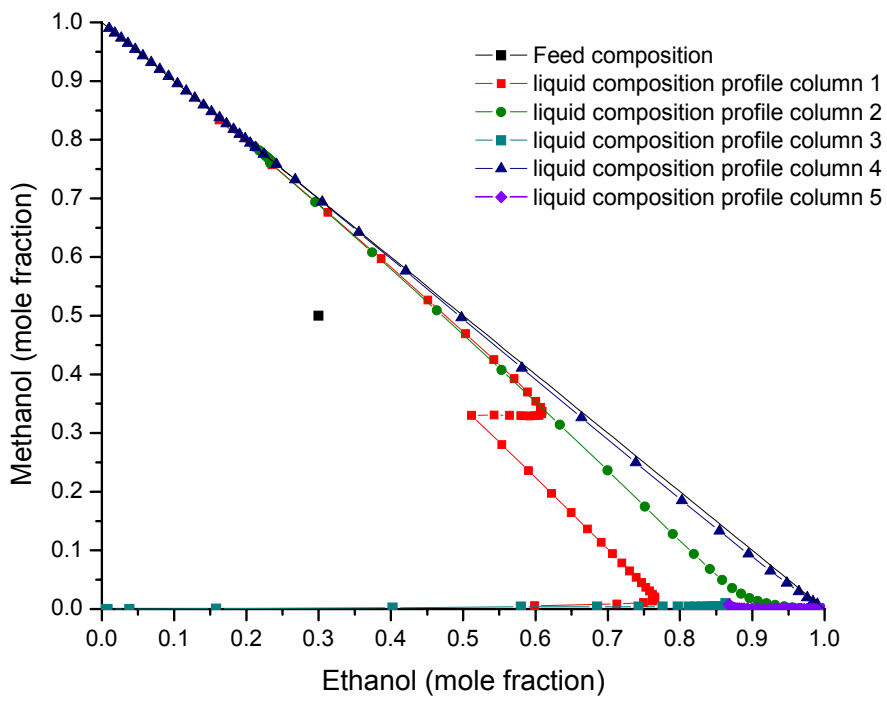

Figure 18: Example 3 - Liquid composition profiles in the preprocessing phase solution 


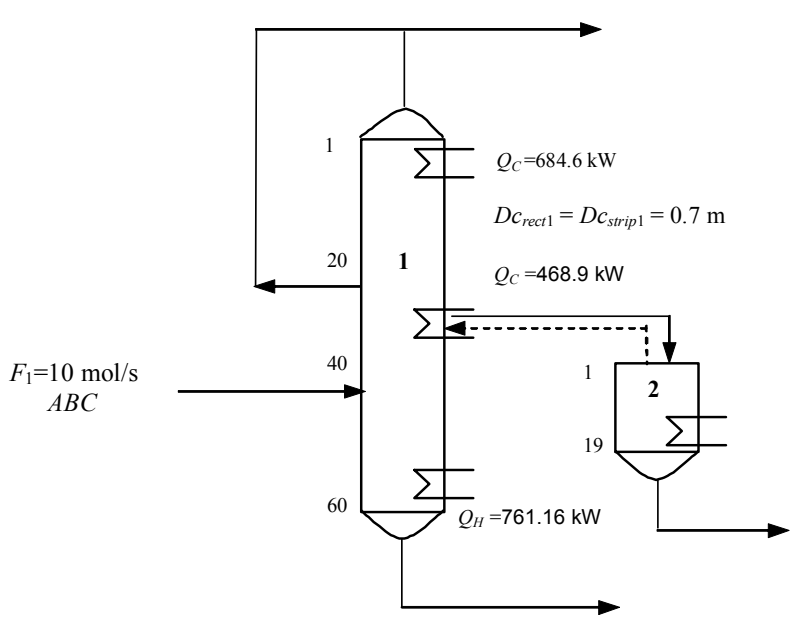

Figure 19: Example 3 - Optimal configuration for products purity of $90 \%$

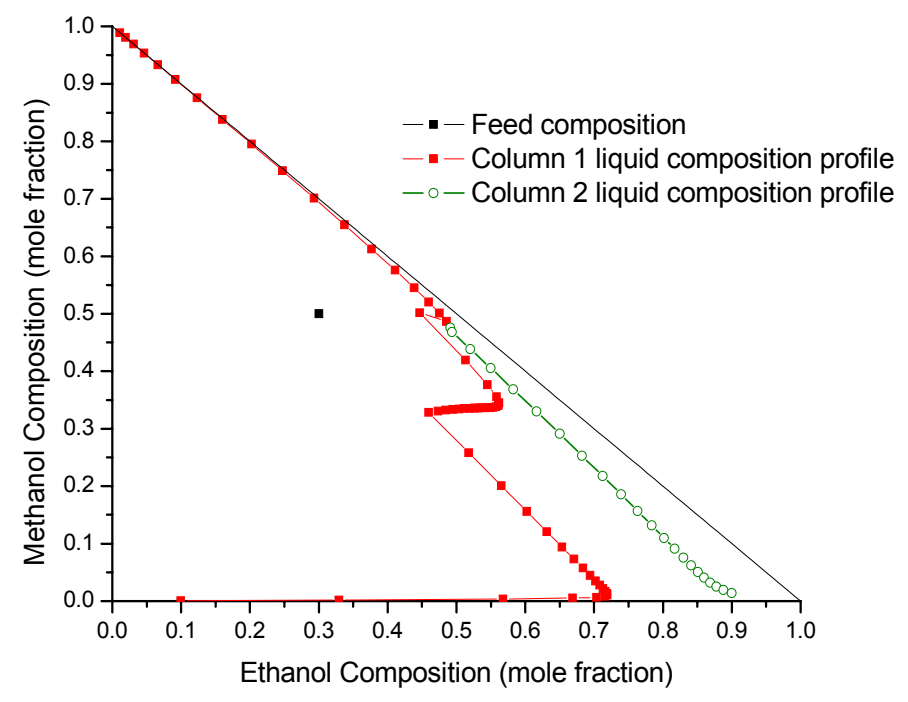

Figure 20: Example 3 - Liquid composition profiles of the economic solution with products purity of $90 \%$ 


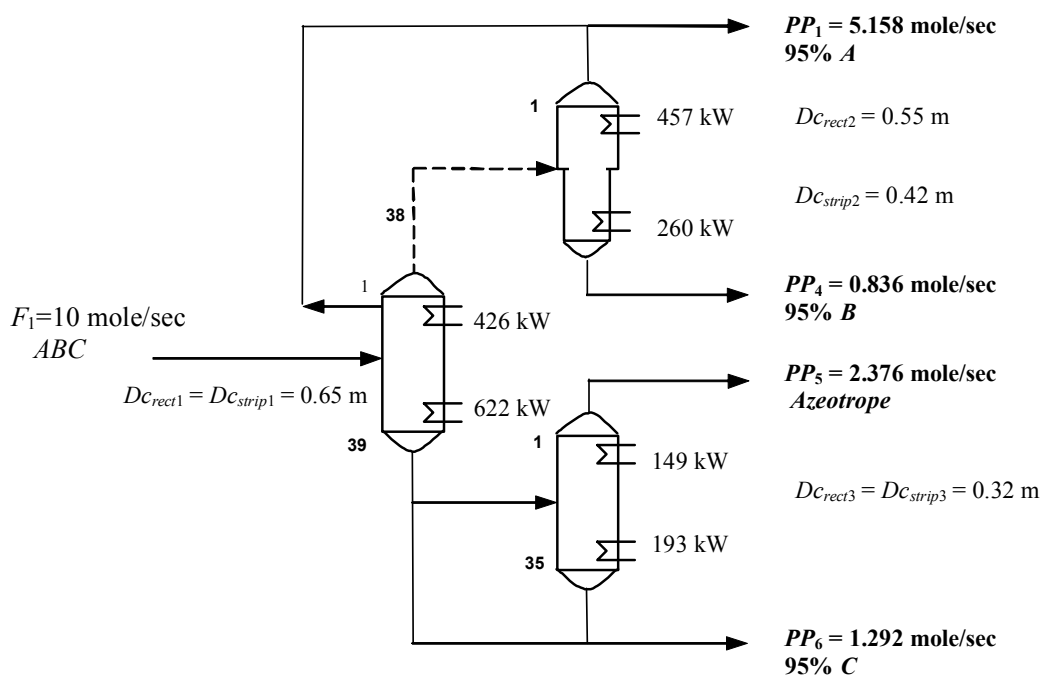

Figure 21: Example 4 - Optimal configuration for products purity of $95 \%$

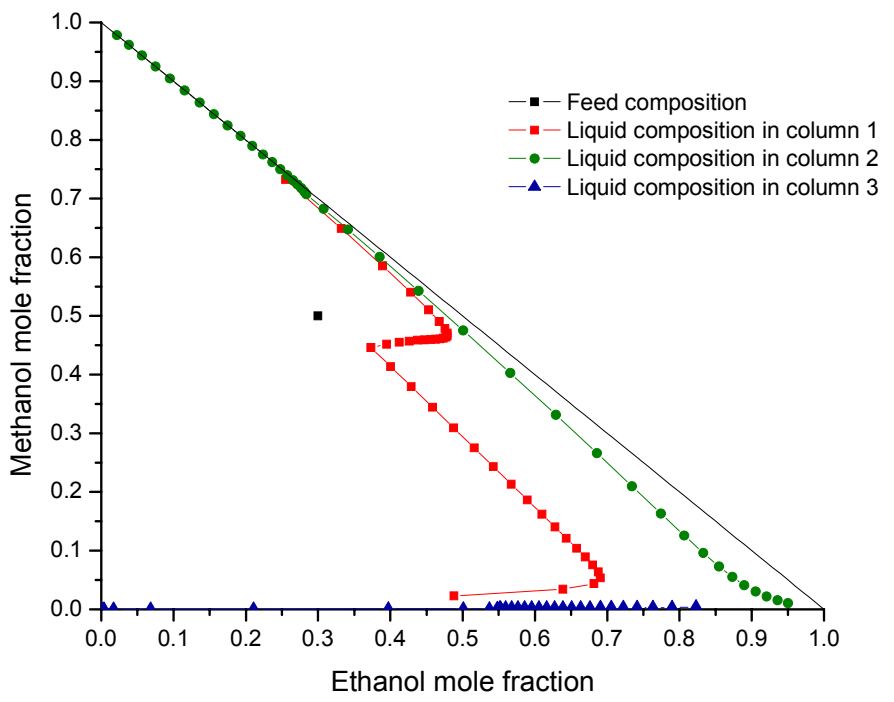

Figure 22: Example 4 - Liquid composition profiles of the economic solution with products purity of $95 \%$ 


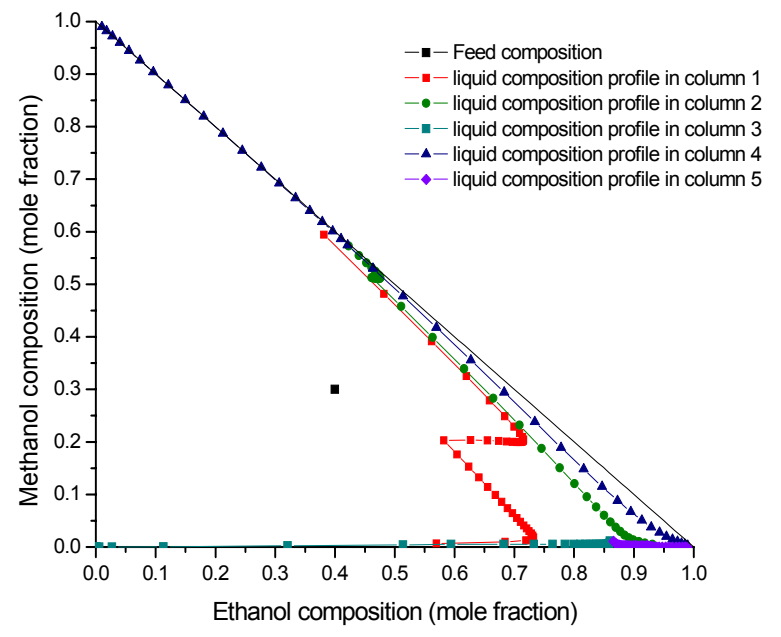

(a)

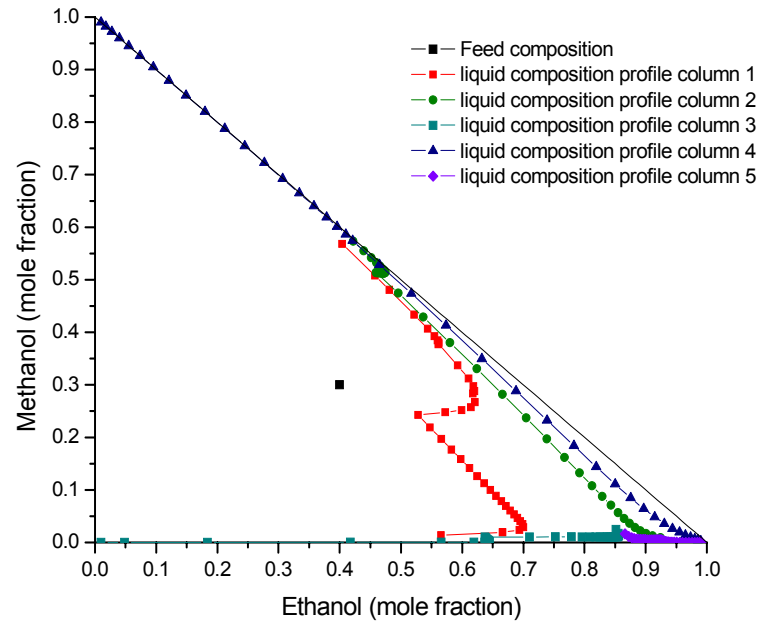

(b)

Figure 23: Preprocessing solutions for feed 0.3, 0.4, 0.3 of methanol, ethanol and water. (a) one condenser. (b) four intercondensers. 
Table 1: Computational results for example 1

\begin{tabular}{ll}
\hline \multicolumn{1}{c}{ Preprocessing Phase: NLP Tray-by-Tray Models } \\
\hline Continuous Variables & 3297 \\
Constraints & 3225 \\
CPU time [min.] & 2.20 \\
\hline \multicolumn{2}{c}{ Model Description } \\
\hline Continuous Variables & 3301 \\
Discrete Variables & 96 \\
Constraints & 3230 \\
Nonlinear nonzero elements & 3244 \\
Number of iterations & 5 \\
NLP CPU time [min.] & 6.97 \\
MILP CPU time [min.] & 2.29 \\
CPU time [min.] & 9.25 \\
\hline Objective Value [\$/year] & $\mathbf{1 4 0 , 8 8 0}$ \\
Total CPU time [min.] & $\mathbf{1 1 . 4 6}$ \\
\hline
\end{tabular}

Table 2: Computational results for example 2

\begin{tabular}{ll}
\hline \multicolumn{1}{c}{ Preprocessing Phase: NLP Tray-by-Tray Models } \\
\hline Continuous Variables & 3297 \\
Constraints & 2831 \\
CPU time [min.] & 1.3 \\
\hline \multicolumn{2}{c}{ Model Description } \\
\hline Continuous Variables & 3301 \\
Discrete Variables & 96 \\
Constraints & 3230 \\
Nonlinear nonzero elements & 3244 \\
Number of iterations & 7 \\
NLP CPU time [min.] & 8.85 \\
MILP CPU time [min.] & 4.22 \\
CPU time [min.] & 13.07 \\
\hline Objective Value [\$/year] & $\mathbf{1 3 7 , 7 6 0}$ \\
Total CPU time [min.] & $\mathbf{1 4 . 3 7}$ \\
\hline
\end{tabular}


Table 3: Computational results for example 3

\begin{tabular}{ll}
\hline \multicolumn{2}{c}{ Preprocessing Phase: NLP Tray-by-Tray Models } \\
\hline Continuous Variables & 9025 \\
Constraints & 8996 \\
CPU time [min.] & 6.05 \\
\hline \multicolumn{2}{c}{ Model Description } \\
\hline Continuous Variables & 8755 \\
Discrete Variables & 210 \\
Constraints & 9466 \\
Nonlinear nonzero elements & 18230 \\
Number of iterations & 3 \\
NLP CPU time [min.] & 54.7 \\
MILP CPU time [min.] & 2.33 \\
CPU time [min.] & 56.1 \\
\hline Objective Value [\$/year] & $\mathbf{3 2 1 , 6 8 0}$ \\
Total CPU time [min.] & $\mathbf{6 2 . 1 5}$ \\
\hline
\end{tabular}

Table 4: Computational results for example 4

\begin{tabular}{ll}
\hline \multicolumn{1}{c}{ Preprocessing Phase: NLP Tray-by-Tray Models } \\
\hline Continuous Variables & 9025 \\
Constraints & 8996 \\
CPU time [min.] & 6.05 \\
\hline \multicolumn{2}{c}{ Model Description } \\
\hline Continuous Variables & 8755 \\
Discrete Variables & 210 \\
Constraints & 9466 \\
Nonlinear nonzero elements & 18230 \\
Number of iterations & 3 \\
NLP CPU time [min.] & 36.26 \\
MILP CPU time [min.] & 3.70 \\
CPU time [min.] & 39.97 \\
\hline Objective Value [\$/year] & $\mathbf{3 1 8 , 4 0 0}$ \\
Total CPU time [min.] & $\mathbf{4 6 . 0 1}$ \\
\hline
\end{tabular}

